\title{
Improved vortex method for large-eddy simulation inflow generation
}

\author{
Baolin Xie ${ }^{a}$, Feng Gao ${ }^{b, *}$, Jérôme Boudet ${ }^{a}$, Liang Shao ${ }^{a}$, Lipeng $\mathrm{Lu}^{\mathrm{c}}$ \\ a Laboratoire de Mécanique des Fluides et d'Acoustique, École Centrale de Lyon, Écully, 69134, France \\ ${ }^{\mathrm{b}}$ Faculty of Engineering and Physical Sciences, University of Surrey, Guildford, GU2 7XH, UK \\ ' School of Energy and Power Engineering, Beihang University, Beijing, 100191, China
}

\section{A R T I C L E I N F O}

\section{Article history:}

Received 24 July 2017

Revised 27 January 2018

Accepted 23 March 2018

Available online 26 March 2018

\section{PACS:}

47.27.ep

\section{Keywords:}

Inflow condition

Vortex method

Large-eddy simulation

Velocity-derivative skewness

\begin{abstract}
A B S T R A C T
The generation of turbulent inflow conditions in large-eddy simulation (LES) is a key ingredient for general applications of LES in both academic turbulent flows and industrial designs with complicated engineering flows. This is because accurate predictions of the fluid behaviour are strongly dependent on the inflow conditions, particularly in turbulent flows at high turbulent Reynolds numbers. This paper aims at improving the vortex method (VM) of Sergent that demands long adaptive distances (12 times the half channel height, for a channel flow at $R e_{\tau}=395$ ) to achieve high quality turbulence, and evaluating the equilibrium of the flow field obtained in terms of both the equilibrium of the mean flow and that of the turbulence (inter-scale turbulent energy transfer). The mean flow equilibrium is checked with classic criteria such as the friction velocity. In order to assess the equilibrium of turbulence, we propose using the velocity-derivative skewness, because it associates with the balance of energy transfer between large- and small-scale fluid motions. Numerical tests with the optimised set of model parameters reveal that the IVM is very efficient, in terms of adaptive distance, in generating high-quality synthetic turbulent fluctuations over a moderate distance: $6 h$ for channel flow and $21 \delta$ for flat-plate boundary layer, with $h$ and $\delta$ being respectively the half channel height and the nominal boundary layer thickness.
\end{abstract}

(c) 2018 Elsevier Ltd. All rights reserved.

\section{Introduction}

Large-eddy simulation (LES) is becoming more and more attractive, owing to its explicit description of large-scale unsteady turbulent motions and the development of sufficient computing power. LES inflow conditions are of great importance as the flow state within the domain of interest is strongly dependent on the inlet conditions, especially for turbulent flows at high turbulent Reynolds numbers [2-5]. In general, the requirements for LES inlet conditions are: (1) being stochastically fluctuating, (2) being spatially and temporally correlated, (3) being qualified by turbulence criteria and (4) being easy to implement.

The LES inflow methods have been reviewed by Keating et al. [6], Tabor et al. [7] and Sagaut et al. [8]. According to these publications, the existing approaches to generate LES inflow conditions can be classified into three major categories: recycling methods, precursor databases, and synthetic turbulence methods. Recycling methods rescale turbulent fluctuations extracted at a downstream position and reintroduce them on the inlet plane, such as the approach of Lund et al. [9]. This imposes a strong spatial correlation

\footnotetext{
* Corresponding author.

E-mail addresses: f.gao@surrey.ac.uk (F. Gao), jerome.boudet@ec-lyon.fr (J. Boudet), liang.shao@ec-lyon.fr (L. Shao).
}

or periodicity to the flow related to a distance between the main inlet and the extraction position. Precursor databases involve performing a separate simulation of a turbulent boundary layer, generating a library to be introduced as inlet condition to the main calculation. This requires large computing costs for high turbulent Reynolds number flows and may impose temporal correlation to the flow due to the finite length of the database. Synthetic turbulence methods aim at directly synthesising turbulence-like fluctuations on the inlet plane of the main computational domain by mathematical functions. However, inappropriate fluctuations at inlet cannot sustain turbulent state and may cause relaminarisation, affecting the main simulation with mispredicted characteristics. Whilst this paper does not attempt to complement the collection of existing LES inflow methods in the review papers mentioned above, it is useful to survey previous studies relevant to the present work.

This paper focuses on the vortex method (VM), a synthetic turbulence approach (also referred to as synthetic eddy method). The conception of this method is to imitate the mechanisms of grid turbulence used in experiments. Perhaps, the earliest attempt to simulate a flow with VM was made by Rosenhead [10]. Further developments of VMs include studies by Maull [11] and Leonard [12]. Their approaches are usually applied to 2D problems, e.g. roll-up of vortex sheets in flows passing bluff bodies. The VM proposed by 
Sergent [1] is based on the Lagrangian form of a 2D vorticity equation. Active vortices calibrated by local turbulent characteristics are injected on a 2D inlet plane to generate turbulent fluctuations over a given mean velocity field, allowing the flow to recover as real turbulence within some adaptive distance. Sergent's VM was later modified by Mathey et al. [13] with a generalised local vortex size described by a turbulent mixing length hypothesis. Mathey et al. [14] incorporated the modified VM in the commercial CFD solver FLUENT, and further applied the VM on problems with more complex geometries. Benhamadouche et al. [15] assessed Sergent's VM for a channel flow at $R e_{\tau}=395$, a pipe flow at $R e_{\tau}=360$ and a backward step flow at $R e_{h}=5100$. They reported the adaptation distance for the channel flow was 12 times the half channel height (h). As a further research of Benhamadouche et al. [15], Jarrin et al. [16] reconstructed turbulent fluctuations on mean velocity fields by means of the Cholesky decomposition of the Reynolds stress tensor, with a finite amount of eddies. Jarrin et al.'s VM can be initialised with mean flow statistics from either a precursor LES or ad-hoc formulae. Further developments of Jarrin's VM considered initial parameters from RANS simulations [17]. Poletto et al. [18] reported assessments of the VMs of Sergent and Jarrin et al. for a channel flow at $R e_{\tau}=395$, and indicated that the adaptive distance of Jarrin et al.'s VM was $10 h$. Poletto et al. also suggested a divergence-free improvement on Jarrin's methodology. Another improvement on Jarrin's VM using a more detailed definition of the eddy shape-function was reported by Pamiès et al. [19]. The VM presents an advantage over other synthetic turbulence methods at a shorter adaptive distance to develop realistic turbulence, as reported by Jarrin et al. [17]. The equilibrium/convergence of mean flows (e.g. friction coefficient $C_{f}$ ) using LES inflow methods have been broadly examined, whereas the equilibrium/convergence of turbulence is rarely evaluated [20].

This study aims at improving the performance, in terms of the adaptive distance, of the original VM developed by Sergent [1], and at introducing the velocity-derivative skewness as a new criterion to evaluate the equilibrium of turbulence. The original VM will be recalled in Section 2, and the methodology to improve the original VM will also be presented. Prior to validating the improved VM (IVM), the commonly used criteria for turbulence qualification will be summarised in Section 3. The velocity-derivative skewness, closely linked to the inter-scale turbulent energy transfer, is presented and introduced as a new criterion to check the state of the simulated turbulence, i.e. whether the spectral equilibrium is established or the turbulence is fully developed. The numerical schemes used in this study will be briefly introduced in Section 4. Next, the validations of the IVM against two canonical test cases will be presented and discussed in Section 5. Finally, the outcomes of this study will be summarised.

\section{Vortex method (VM)}

Physically, the VM could be considered as a counterpart of an active grid turbulence generator used in experiments, aiming at triggering turbulent fluctuations at the inlet of the computational domain for unsteady simulations. Vortices injected into the inlet plane by the VM mimic those obtained with active grids used in laboratory experiments for generating turbulent flows. One should note that the VM only generates velocity fluctuations within the inlet plane, i.e. fluctuations are not yielded for the velocity component in the normal direction to the inlet plane. Theoretically, the $\mathrm{VM}$ is based on the Lagrangian form of the 2D vorticity equation.

$\frac{\partial \omega}{\partial t}+(\boldsymbol{u} \cdot \nabla) \omega=v \nabla^{2} \omega$

The velocity vector $\boldsymbol{u}$ is,

$\boldsymbol{u}=\nabla \times \boldsymbol{\psi}+\nabla \phi$
The vorticity vector is the curl of the velocity vector.

$\boldsymbol{\omega}=\nabla \times \boldsymbol{u}$

with $\boldsymbol{\psi}=(\psi, 0,0)$ and $\boldsymbol{\omega}=(\omega, 0,0) . \psi$ is the $2 \mathrm{D}$ stream function and $\phi$ is the velocity potential. Thus the vorticity component along the streamwise direction can be expressed as:

$\omega=-\nabla^{2} \psi$

The velocity vector at a location $\boldsymbol{x}$ induced by a general shape vortex centred at $\boldsymbol{x}^{\prime}$ is given by the Biot-Savart law:

$\boldsymbol{u}(\boldsymbol{x})=-\frac{1}{2 \pi} \iint_{R^{2}} \frac{\left(\boldsymbol{x}-\boldsymbol{x}^{\prime}\right) \times \omega\left(\boldsymbol{x}^{\prime}\right) \cdot \boldsymbol{n}}{\left|\boldsymbol{x}-\boldsymbol{x}^{\prime}\right|^{2}} d \boldsymbol{x}^{\prime}$

where $\boldsymbol{n}$ is the unit vector aligned with the positive direction of the vortex's rotational axis.

In practice, the vorticity at a location $\boldsymbol{x}$ is a superposition of vorticities induced by a number of vortices. If $N$ is the number of vortices injected on the plane and each vortex has its own circulation $\Gamma_{i}$ and spatial distribution $\xi_{i}$, the vorticity at this location is expressed as:

$\omega(\boldsymbol{x}, t)=\sum_{i=1}^{N} \Gamma_{i} \xi_{i}\left(\left|\boldsymbol{x}-\boldsymbol{x}_{i}\right|, t\right)$

with $i$ denoting the vortex index and $\boldsymbol{x}_{i}$ being the coordinate of its centre. The spatial distribution $\xi$ uses a modified Gaussian shape [21]:

$\xi(\boldsymbol{x})=\frac{1}{2 \pi \sigma^{2}}\left(2 e^{-\frac{|\boldsymbol{x}|^{2}}{2 \sigma^{2}}}-1\right) e^{-\frac{|\boldsymbol{x}|^{2}}{2 \sigma^{2}}}$

where $\sigma$ is the radius of the vortex.

Therefore, the velocity vector at $\boldsymbol{x}$ induced by a vortex field is expressed by:

$\boldsymbol{u}(\boldsymbol{x})=-\frac{1}{2 \pi} \sum_{i=1}^{N} \Gamma_{i} \frac{\left(\boldsymbol{x}-\boldsymbol{x}_{i}\right) \times \boldsymbol{n}}{\left|\boldsymbol{x}-\boldsymbol{x}_{i}\right|^{2}}\left(1-e^{-\frac{\left|\boldsymbol{x}-\boldsymbol{x}_{i}\right|^{2}}{2 \sigma_{i}^{2}}}\right) e^{-\frac{\left|\boldsymbol{x}-\boldsymbol{x}_{i}\right|^{2}}{2 \sigma_{i}^{2}}}$

This formula gives a steady velocity field determined by a vortex field. In order the get a fluctuating velocity field for unsteady simulations, the vortices should displace and inverse directions over time to mimic the Brownian motion. Now, the parameters of the VM formulation are listed as follows:

$N$ : Total number of vortices injected on the inlet plane.

$\sigma$ : Radius of a vortex.

$\Gamma$ : Circulation of a vortex.

$\lambda$ : Free path of a vortex: the distance travelled by a vortex between successive changes in moving direction.

$\Delta \tau$ : Vortex reversal time: a vortex may inverse its direction after each reversal time $\Delta \tau$.

\subsection{Original $V M$}

This section introduces the VM of Sergent [1] and Mathey et al. [13]. The inflow condition is initialised by RANS type data whatever they are from a RANS calculation or are derived from the statistics of an LES simulation. The unsteady inflow is generated by the VM at each iteration. The velocity fluctuations are only generated for the two components within the inlet plane, and no fluctuation is imposed for the component in the normal direction to the inlet plane. The choices of VM parameters are explained separately.

\section{Total vortex number $\mathrm{N}$}

Sergent [1] conducted tests with different vortex numbers $N$ and found that results were independent of $N$ within the test matrix $(N \in[100,300])$ performed on channel flow. 
Vortex radius $\sigma$

Originally, the vortex radius $\sigma$ of Sergent's VM was set to an adhoc value throughout the injection plane. Mathey et al. generalised the local vortex radius through a turbulent mixing length hypothesis. The vortex radius is computed by the mean turbulent kinetic energy and turbulent dissipation rate imposed on the inlet plane:

$\sigma=\frac{c k^{3 / 2}}{2 \varepsilon}$

where $c=C_{\mu}^{3 / 4}$ with $C_{\mu}=0.09$. The minimum value of $\sigma$ is bounded by the local grid size to ensure that the vortices are always in the resolved scales.

Vortex circulation $\Gamma$

The circulation $\Gamma$ of the VM is determined by the root mean square of the local velocity fluctuations $\boldsymbol{U}_{\text {rms }}^{\prime}$. Given $N$ the number of vortices and $S$ the area of the injection plane, the circulation $\Gamma$ can be expressed as follows:

$\Gamma(\boldsymbol{x})=\sqrt{\frac{4 \pi S}{N(2 \ln 3-3 \ln 2)}} \boldsymbol{U}_{\mathrm{rms}}^{\prime}(\boldsymbol{x})$

where $\boldsymbol{U}_{\mathrm{rms}}^{\prime}=\sqrt{\left\langle v^{\prime} v^{\prime}\right\rangle+\left\langle w^{\prime} w^{\prime}\right\rangle}$ only includes the two fluctuating velocity components within the injection plane.

Taking the isotropic turbulence hypothesis that $\left\langle u^{\prime} u^{\prime}\right\rangle=\left\langle v^{\prime} v^{\prime}\right\rangle=$ $\left\langle w^{\prime} w^{\prime}\right\rangle=\frac{2}{3} k$, the circulation $\Gamma$ is given as:

$\Gamma(\boldsymbol{x})=4 \sqrt{\frac{\pi S k(\boldsymbol{x})}{3 N(2 \ln 3-3 \ln 2)}}$

Vortex free path $\lambda$

In the VM, vortices displace randomly within the injection plane at a velocity of $U_{d}$ to mimic the Brownian motion. Sergent has carried out numerical simulations with $U_{d}$ being equal to $1 \%$, $5 \%$ and $10 \%$ of the inlet bulk velocity $U_{b}$. Amongst these three tests, $U_{d}=5 \% U_{b}$ gave the best comparison with DNS data. In this approach, a vortex changes its moving direction between two adjacent time steps. So the free path of the vortices $\lambda$ can be expressed by Eq. (12). This value was also used in the studies of Mathey et al. In Sergent's VM, two kinds of treatments have been tested when a vortex touches a solid wall. One is that vortices are destroyed when they reach solid walls and the same number of vortices is reinjected on the inlet plane. The other is that vortices bounce back as they touch the solid walls. The latter method was preferred as it provides the conservation of vortex density along the wall normal direction. This treatment was not considered in the studies of Mathey et al.

$\lambda=5 \% U_{b} d t$

\section{Vortex reversal time $\Delta \tau$}

The parameter $\Delta \tau$ represents a time interval by the end of which vortices may inverse randomly their rotational directions. In Sergent's work, $\Delta \tau$ is defined with some ad-hoc values. Mathey et al. introduced a more meaningful formulation for $\Delta \tau$ based on the turbulent time scale:

$\Delta \tau=\frac{k}{\varepsilon}$

Through a series of systematic studies, Sergent suggests that the most important parameters in the VM are the vortex radius $\sigma$ and the vortex displacement velocity $U_{d}$. In addition, a better convergence can be achieved by increasing the number of vortices $N$. However, the turbulent fluctuations generated by the original VM need more evaluations, such as energy balance, to confirm their qualification.
Treatment of boundary conditions

Regarding wall boundary conditions, ghost vortices are used to compensate the high fluctuating velocity components induced by the real vortices, letting the velocity gradually reduce to zero towards no-slip walls. Similar ghost vortices are applied to ensure continuity across periodic boundaries. More details can be found in Refs. [1,15].

\subsection{Improved VM (IVM)}

In order to generalise the VM, especially for applications with complex geometries, the VM parameters need to be determined by local turbulence characteristics rather than ad-hoc values employed in the original VM. Another issue is that the fluctuating flow field generated is usually not spatially and temporally correlated as for real turbulence, increasing the adaptive distance. In the present study, rather than paying attention to the synthesised inlet flow field, efforts are made on reducing the adaptive distance necessary to get accurate statistics. As for the VM, the IVM only generates velocity fluctuations for the components within the inlet plane, and no fluctuation is created for the third component. Improvements of the VM are made on four out of the five parameters, except the vortex number $N$ that has been well studied by Sergent [1].

\section{Vortex radius $\sigma$}

From a point of view of the energy cascade, large-scale eddies are responsible for the containing and transferring of energy. Inspired by Mathey et al., the size of these eddies (vortex radius) should be comparable with the energy-containing length scale:

$\sigma \sim L$

In the vortex radius formulation for the IVM, $\sigma$ is calculated from the Kolmogorov scale $\eta$ that characterises the smallest eddies.

$\eta \equiv\left(v^{3} / \varepsilon\right)^{1 / 4}$

In order to find out the relation between the Kolmogorov scale $\eta$ and the energy-containing length scale $L$, one should consider homogeneous isotropic turbulence where the turbulent dissipation rate writes:

$\varepsilon \sim u_{L}^{3} / L$

with $u_{L}$ being the characteristic velocity of the largest eddies.

Inserting Eq. (16) into Eq. (15), the energy-containing length scale can be expressed as:

$L \sim R e_{t}^{3 / 4} \eta=\operatorname{Re}_{t}^{3 / 4}\left(v^{3} / \varepsilon\right)^{1 / 4}$

where $R e_{t}=u_{L} L / v$ is the turbulent Reynolds number.

Therefore, a new formulation for the vortex radius $\sigma$ is proposed in Eq. (18), which relies on the Kolmogorov scale $\eta$ and the turbulent Reynolds number $R e_{t}$.

$\sigma=C_{1} \operatorname{Re}_{t}^{3 / 4}\left(v^{3} / \varepsilon\right)^{1 / 4}$

with $C_{1}$ a coefficient to be tuned.

For practical reasons in programming and result analysis, a nondimensional form of $\sigma$ is given below, since the Navier-Stokes equations are usually solved in non-dimensional form.

$\sigma^{+}=\sigma / L=C_{1}\left(\left(v^{+}\right)^{3} / \varepsilon^{+}\right)^{1 / 4}$

where $v^{+}=1$ if $v$ itself is used as the reference value for normalisation, and $\varepsilon^{+}=\varepsilon /\left(u_{L}^{3} / L\right)$ can be directly calculated with the nondimensional flow solution as the simulation is running. 


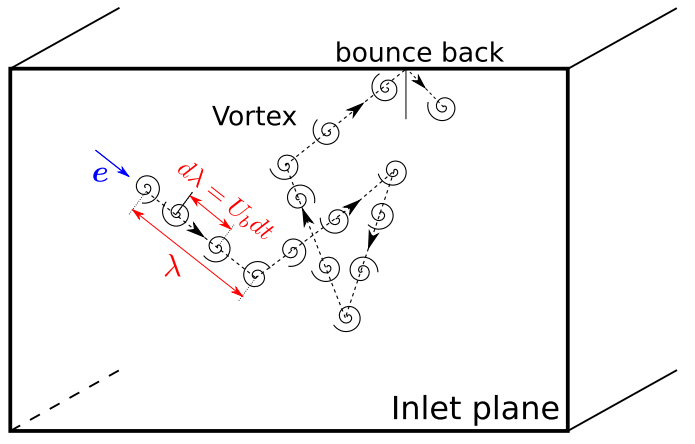

Fig. 1. Sketch of the enhanced vortex displacement.

Vortex free path $\lambda$

In the VM of Sergent and Mathey et al., the vortices move randomly on the inlet plane with a displacement speed associated with the inlet bulk velocity $U_{b}$, and change their moving directions between two adjacent time steps. Thus the vortex free path is the distance a vortex travels per time step $d t$, as expressed in Eq. (12). However numerical tests reveal that most vortices just oscillate around their initial positions with very small oscillating amplitudes.

Therefore, an enhanced vortex displacement pattern is introduced for the IVM, as illustrated in Fig. 1. It consists in adding a memory time to the vortex displacement. For each time interval equal to the time step $d t$, a vortex moves its position within the inlet plane along a certain direction $\boldsymbol{e}$ with a step length $d \lambda$, as suggested in Eq. (19). But this vortex will hold its moving direction until the distance it travelled along the direction $\boldsymbol{e}$ reaches its free path $\lambda$. Note that the ad-hoc value of $5 \%$ assigned to the vortex displacement speed in the VM of Sergent is eliminated in the IVM formulation. The vortex free path $\lambda$ should have an order of magnitude similar to the vortex radius $\sigma$ and be much larger than $d \lambda$. The formulation of $\lambda$ for the enhanced vortex displacement is given in Eq. (20), with a coefficient $C_{3}$ to be determined in different test cases. This strategy ensures that a vortex is active enough in moving its position rather than just oscillating around its original location. The vortices bounce back as they reach solid walls.

$d \lambda=U_{b} d t$

$\lambda=C_{3} \sigma$

\section{Vortex circulation $\Gamma$}

In order to control the intensity of the fluctuations, an additional coefficient is added to the original VM's circulation formula, as given below:

$\Gamma=C_{2} \Gamma_{0}=4 C_{2} \sqrt{\frac{\pi S k}{3 N(2 \ln 3-3 \ln 2)}}$

\section{Vortex reversal time $\Delta \tau$}

In the IVM, the vortex reversal time $\Delta \tau$ follows Eq. (13) suggested by Mathey et al. based on the turbulence time scale. Also, a coefficient $C_{4}$ is added to the equation to be tuned with different test cases.

$\Delta \tau=C_{4} \frac{k}{\varepsilon}$

\section{Turbulence qualification criteria}

As mentioned in Introduction, the fluctuations generated by LES inlet conditions must lead to high-quality turbulence within a short adaptive length. Here, high quality means fully developed turbulence where the spectral inter-scale energy transfer is in equilibrium. A number of LES inflow methods has proved good convergence/equilibrium of the mean flow fields obtained, but the equilibrium of turbulence was rarely accessed [20]. Prior to validating the IVM against test cases, the available criteria for qualifying turbulent flows are summarised in this section. Then the proposition of the velocity-derivative skewness as a new criterion for turbulence qualification will be elaborated. Those criteria will be used to evaluate the turbulence generated by the IVM in Section 5.

\subsection{Existing criteria}

The existing turbulence qualification criteria can be classified into two categories. One is evolutionary criteria: this sort of criteria evaluates the state of the flow along a certain direction (usually streamwise). The other is local criteria, which examine the flow behaviour at a certain location.

\section{Evolutionary criteria}

The skin friction coefficient $C_{f}$ is the wall friction normalised by the free-stream dynamic head. It writes:

$C_{f}=\frac{\tau_{w}}{\frac{1}{2} \rho U_{\infty}^{2}}$

where $\tau_{w}$ is the local wall shear stress, $\rho$ is the fluid density and $U_{\infty}$ the free-stream velocity.

For a given flow with a fixed reference velocity, $C_{f}$ in a turbulent boundary layer is larger than in a laminar boundary layer. In a laminar boundary layer over a flat plate, the relation between $C_{f}$ and $R e_{x}$ is given by Eq. (24) (solution of Blasius equation) [22]. In turbulent regime, Michel's empirical relation [23] applies, as expressed in Eq. (25).

$C_{f}=0.664 R e_{x}^{-1 / 2}$

$C_{f}=0.0368 R e_{x}^{-1 / 6}$

The shape factor $H_{12}$ is the ratio between the displacement thickness $\delta_{1}$ and the momentum thickness $\delta_{2}$ of the boundary layer.

$H_{12}=\frac{\delta_{1}}{\delta_{2}}$

where $\delta_{1}=\int_{0}^{\infty}\left(1-\frac{u(y)}{U_{\infty}}\right) d y$, and $\delta_{2}=\int_{0}^{\infty} \frac{u(y)}{U_{\infty}}\left(1-\frac{u(y)}{U_{\infty}}\right) d y$ in incompressible form.

Generally, the shape factor $H_{12}$ is close to 2.6 for a laminar boundary layer (Blasius solution) and decreases to about $1.3 \sim 1.4$ for a turbulent boundary layer [22].

\section{Local criteria}

Most commonly, a turbulent boundary layer profile at a given turbulent Reynolds number should obey the law of the wall, which includes viscous $\left(y^{+}<5\right)$, buffer $\left(5<y^{+}<30\right)$ and log-law layers. This can be assessed by Eq. (27) (for the viscous layer) and Eq. (28) (for the log layer).

$u^{+}=y^{+}$

$u^{+}=\frac{1}{\kappa} \ln \left(y^{+}\right)+B$

where $u^{+}=u / u_{\tau}$ is velocity scaled by the friction velocity $u_{\tau}$, and $y^{+}=y u_{\tau} / \nu$ the distance in wall units. $\kappa \approx 0.41$ is von Karman's constant, and $B \approx 5.2$.

The mean velocity profile, as well as the profiles of Reynolds stresses, can also be compared to existing experimental and DNS data at a similar turbulent Reynolds number. 
LES directly solves the large-scale fluid motions and models the small-scale ones. If the turbulent Reynolds number is sufficiently high, resolved-scale motions issued from a correct LES simulation should capture some basic spectral characteristics such as the $-5 / 3$ slope of turbulent kinetic energy spectra.

\subsection{Velocity-derivative skewness}

In addition to the convergence/equilibrium of the mean flow, which can be assessed by the criteria mentioned above, the equilibrium of turbulence must be verified as well, for the flow field generated by a synthetic inflow method. In turbulent flows, energy is transferred from large-scale eddies to small-scale eddies and is finally dissipated by the smallest eddies, according to the energy cascade. There is also a back-scatter phenomenon that transports energy from small-scale eddies to large-scale eddies. The energy transfer between the large-scale and small-scale fluid motions must be balanced in a fully developed turbulence. A simple indicator of this balance or equilibrium of fully developed turbulence could be the velocity-derivative skewness.

A number of studies [24-27] suggests the close connection between the velocity-derivative skewness and the transfer of energy between different scales. This relation can be derived following their publications, which are briefly summarised as follows.

The second-order and third-order longitudinal structure functions are given in Eqs. (29) and (30) for isotropic turbulence [27]:

$D_{L L}(r)=\frac{2}{15} r^{2} \int \kappa^{2} E(\kappa) d \kappa$

$D_{L L L}(r)=-\frac{2}{35} r^{3} \int \kappa^{2} T(\kappa) d \kappa$

where $E(\kappa)$ and $T(\kappa)$ denote the energy spectrum and the nonlinear transfer, respectively. $\kappa$ is the wave number.

The velocity-increment skewness is then defined as:

$S(r)=\frac{D_{L L L}(r)}{D_{L L}(r)^{\frac{3}{2}}}$

For very small scale $r$, inserting Eqs. (29) and (30) in Eq. (31), the velocity-derivative skewness $S_{k}$ can be expressed as:

$S_{k}=\lim _{r \rightarrow 0} S(r)=\frac{\left\langle(\partial u / \partial x)^{3}\right\rangle}{\left\langle(\partial u / \partial x)^{2}\right\rangle^{\frac{3}{2}}}=-\frac{15^{\frac{3}{2}}}{35 \sqrt{2}} \frac{\int_{0}^{\infty} \kappa^{2} T(\kappa) d \kappa}{\left[\int_{0}^{\infty} \kappa^{2} E(\kappa) d \kappa\right]^{\frac{3}{2}}}$

Eq. (32) indicates that $S_{k}$ directly associates with the energy transfer. Considering situations at high Taylor-scale Reynolds numbers $\left(R e_{\lambda}\right)$, if the non-stationarity is negligible at high wave number $\kappa$, or if the turbulence keeps stationary with a forcing term only active at small $\kappa$, there is a relation between $E(\kappa)$ and $T(\kappa)$ :

$\int \kappa^{2} T(\kappa) d \kappa \approx \int 2 \nu \kappa^{4} E(\kappa) d \kappa$

thus,

$S_{k}=\frac{\left\langle(\partial u / \partial x)^{3}\right\rangle}{\left\langle(\partial u / \partial x)^{2}\right\rangle^{\frac{3}{2}}} \approx-\frac{15^{\frac{3}{2}} \sqrt{2} v}{35} \frac{\int_{0}^{\infty} \kappa^{4} E(\kappa) d \kappa}{\left[\int_{0}^{\infty} \kappa^{2} E(\kappa) d \kappa\right]^{\frac{3}{2}}}$

$S_{k}$ is a function of the Taylor-scale Reynolds number of the flow, as $E(\kappa)$ depends on it. For a turbulent flow at a given Taylorscale Reynolds number, the transfer of energy from large scales to small scales is balanced by the dissipation rate, since the process of the energy cascade is established or turbulence is fully developed. Therefore, $S_{k}$ is a negative value at a certain level depending on the Taylor-scale Reynolds number.

Some other studies also show evidences that $S_{k}$ is constant but dependent on the Taylor-scale Reynolds number, as summarised in Table 1.
Therefore, we recommend using the velocity-derivative skewness $S_{k}$ as a new criterion to assess turbulent flows obtained by LES, since it provides a possible measurement of spectral energy balance or the non-linear inter-scale interaction of resolved scales. A general three-dimensional (3-D) formula of the velocityderivative skewness is given in Eq. (35):

$S_{k}^{*}=\frac{\left\langle\left(\partial u_{i} / \partial x_{j}\right)^{3}\right\rangle}{\left\langle\left(\partial u_{i} / \partial x_{j}\right)^{2}\right\rangle^{\frac{3}{2}}}$

In the present study, for simplicity and without losing pertinence of the approach, only the longitudinal velocity-derivative skewness given in Eq. (36) is used.

$S_{k}=\frac{\left\langle(\partial u / \partial x)^{3}\right\rangle}{\left\langle(\partial u / \partial x)^{2}\right\rangle^{\frac{3}{2}}}$

\section{Numerical schemes}

The numerical simulations have been carried out with the in-house solver Turb'Flow, developed in LMFA ${ }^{1}$. This code solves compressible Navier-Stokes equations using vertex-centred finitevolume discretisation on multi-block structured grids, aiming at simulating complex flows in turbomachinery. Validations of this solver will be given in Section 5, and other validations on corner separation and tip leakage flows can be found in Refs. [3,4].

LES is adopted in this study, where the large-scale fluid motions are directly resolved and the sub-grid scale (SGS) fluid motions are modelled. The mesh is considered to act as a box filter that separates fluid behaviours from different scales. The WALE (Wall-Adapting Local Eddy-viscosity) model proposed by Nicoud and Ducros [34] is used to predict the SGS behaviours with a model constant $C_{w}=0.5$. The filter size $\Delta$ is given by the grid cell spacing based on the cube root of the control volume.

The inviscid fluxes are discretised with a 4-point centred scheme, and the viscous fluxes are interpolated with a 2-point centred scheme. A $4^{\text {th }}$-order artificial viscosity is employed, inspired by Jameson [35], to eliminate spurious grid-to-grid oscillations. An explicit five-stage Runge-Kutta scheme with a global constant time step is used for temporal discretisation.

\section{Numerical results and discussions}

The IVM is tested on channel flows at two different turbulent Reynolds numbers, and on a flat-plate boundary layer. All the five IVM parameters and the associated constants are investigated. Results are validated and discussed.

\subsection{Channel flow}

The IVM is at first validated and tuned with channel flow test cases [36]. An LES of a periodic channel flow at $R e_{\tau}=395$ has been carried out and is taken as reference for parametric tuning of the IVM and result comparison. A RANS simulation of the same channel have been conducted to provide information, such as profiles of mean velocity, turbulent kinetic energy, and turbulent dissipation rate. This information is then used on the inlet plane of the channel to initiate the IVM simulation. The constants obtained from the channel flow at $R e_{\tau}=395$ are applied to another channel flow at a higher turbulent Reynolds number $\left(R e_{\tau}=590\right)$.

\subsubsection{Periodic channel flow at $\operatorname{Re}_{\tau}=395$}

The dimensions of the periodic channel flow test case are $2 \pi h \times 2 h \times \pi h$ in the streamwise, wall-normal and spanwise directions, respectively. The LES mesh, consisting of $49 \times 89 \times 41$ points,

\footnotetext{
${ }^{1}$ Laboratoire de Mécanique des Fluides et d'Acoustique, École Centrale de Lyon
} 
Table 1

Experimental and numerical values of the velocity-derivative skewness.

\begin{tabular}{lll}
\hline Ref. & Data & $S_{k}$ \\
\hline$[28]$ & Channel flow experiment & $S_{k} \in[-0.4,-0.3]$ throughout the channel $S_{k} \approx-0.8$ near the wall \\
{$[29]$} & Homogeneous isotropic turbulence & $S_{k}$ is constant and is dependent on $\operatorname{Re} e_{\lambda}$ \\
{$[30]$} & Data of Antonia et al. [31], $\operatorname{Re} e_{\lambda}>400$ & $S_{k} \approx-0.5\left(\operatorname{Re} e_{\lambda} / 400\right)^{0.11} \approx-0.26 R e_{\lambda}^{0.11}$ \\
{$[32]$} & Wind tunnel experiment & $S_{k} \approx-0.33 R e_{\lambda}^{0.09}$ \\
{$[33]$} & Direct numerical simulation & $S_{k} \approx-(0.32 \mp 0.02) R e_{\lambda}^{0.11 \pm 0.03}$ \\
\hline
\end{tabular}

Table 2

Comparison of the channel flow friction velocity $u_{\tau}$.

\begin{tabular}{llll}
\hline & DNS [36] & LES & RANS \\
\hline$u_{\tau}[\mathrm{m} / \mathrm{s}]$ & 0.590 & 0.595 & 0.536 \\
Error $\left(\frac{u_{\tau}-u_{\tau, D N S}}{u_{\tau, D N S}}\right)[-]$ & N.A. & $+0.85 \%$ & $-9.15 \%$ \\
\hline
\end{tabular}
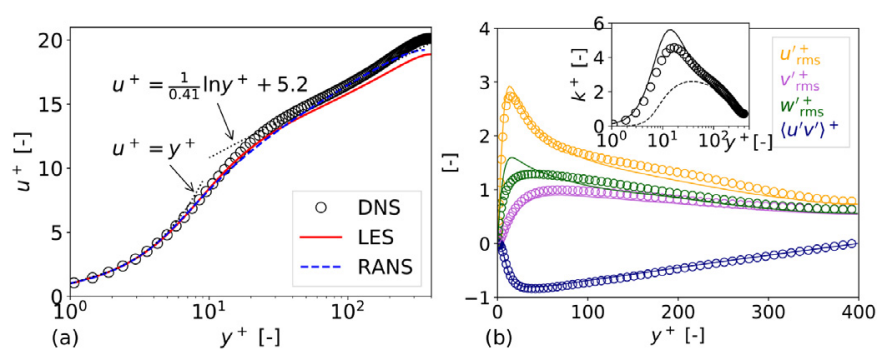

Fig. 2. Comparison of channel flow results at $R e_{\tau}=395$, between the DNS of Kim et al. [36], LES and RANS. (a) mean velocity profile, (b) velocity fluctuations.

is uniform in both the streamwise and spanwise directions and follows a tanh distribution in the wall-normal direction. This yields grid resolutions in wall units in the streamwise, wall-normal and spanwise directions: $\Delta x^{+}=52, \Delta y^{+}=0.5$ and $\Delta z^{+}=31$. No-slip adiabatic wall conditions are applied on the upper and lower walls of the channel, and the boundaries of the other two directions are linked by periodic conditions. The flow is driven by a source term added to the momentum and energy equations, maintaining the mass flow rate in the streamwise direction. A dimensional time step of $1 \times 10^{-7} \mathrm{~s}$ is used in the LES simulations, corresponding to a Courant number (under the CourantFriedrichsLewy condition) of 2.8. A standalone RANS simulation has been conducted on the same grid $^{2}$, using the standard Wilcox $k-\omega$ model (1988) [37] to close the RANS equations.

A comparison of the friction velocity is given in Table 2 for a dimensional half channel height $h=0.01 \mathrm{~m}$. The LES with the WALE SGS model slightly over-predicts $u_{\tau}$, while RANS under-estimates $u_{\tau}$ by $9 \%$. The comparisons of the channel flow mean velocity and velocity fluctuation profiles between the DNS of Kim et al. [36], LES, and RANS are shown in Fig. 2. A good agreement on the mean velocity profiles is observed between the three simulations. The LES gives very good predictions of the components $u_{\mathrm{rms}}^{\prime+}, v_{\mathrm{rms}}^{\prime+}$, $u^{\prime} v^{\prime}$, and also shows a reasonably good comparison on $w_{\mathrm{rms}}^{\prime+}$ in terms of the peak location and amplitude level. The RANS model yields $\left\langle u^{\prime} u^{\prime}\right\rangle=\left\langle v^{\prime} v^{\prime}\right\rangle=\left\langle w^{\prime} w^{\prime}\right\rangle=\frac{2}{3} k$, with a $50 \%$ under-estimate of the turbulent kinetic energy $k$.

\subsubsection{Channel flow LES at $\operatorname{Re}_{\tau}=395$ with IVM: parametric tuning}

The IVM is applied to the same channel flow as discussed above. The upstream boundary is set as inlet where the IVM is initiated based on the RANS solution obtained previously. The downstream boundary is defined as outlet using a pressure boundary condition mixed with a non-reflective condition, allowing a partial

\footnotetext{
2 For practical reasons, the RANS simulation was performed in 3-D. A twodimensional (2-D) RANS would be sufficient.
}

Table 3

Run list for the coefficient calibration of the vortex radius $\sigma$.

\begin{tabular}{lllll}
\hline Test case & $C_{1}$ in $\sigma$ & $C_{2}$ in $\Gamma$ & $C_{3}$ in $\lambda$ & $C_{4}$ in $\Delta \tau$ \\
\hline TC1a & 1 & 1 & $\lambda=d \lambda$ & 1 \\
TC1b & $1 / 2$ & 1 & $\lambda=d \lambda$ & 1 \\
TC1c & $1 / 4$ & 1 & $\lambda=d \lambda$ & 1 \\
TC1d & $1 / 16$ & 1 & $\lambda=d \lambda$ & 1 \\
\hline
\end{tabular}

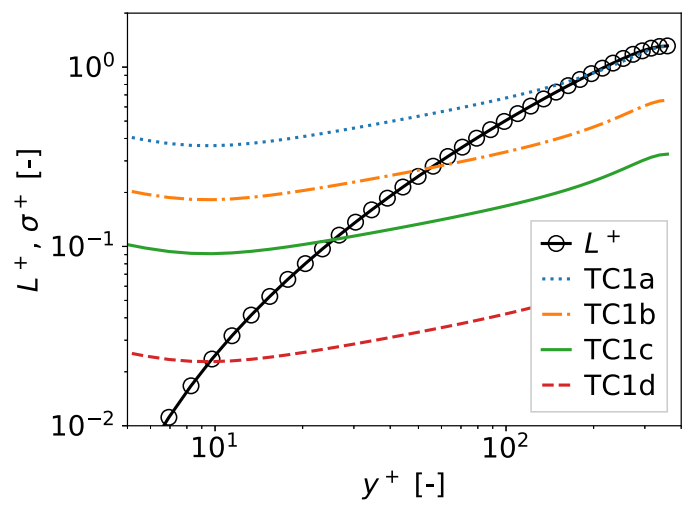

Fig. 3. Comparison of $L^{+}$and $\sigma^{+}$obtained from the RANS channel flow solution.

evacuation of spurious acoustic waves. The other boundaries keep the same conditions as for the periodic channel flow. The effect of the number of vortices have been already investigated by Sergent [1]. Since the IVM is only applied on a 2-D plane, the computation cost increase with the number of vortices is marginal at todays computing capability. The total number of vortices injected on the inlet plane is set to 800 .

The constants of the IVM are calibrated separately and systematically using the periodic LES data, in terms of the $C_{f}$ evolution, and the mean velocity and velocity fluctuation profiles. An appropriate choice of the IVM constants must allow the $C_{f}$ to recover approximately its original value within a short adaptive distance and must allow the mean velocity and velocity fluctuation profiles to agree as well as possible with the periodic LES data. Finally, the evolution of $S_{k}$ is introduced for the results with the best choice of the constants.

\section{Vortex radius $\sigma$}

As already discussed, the vortex radius $\sigma$ should be comparable with the turbulence energy-containing scale $L$, which provides a guide for choosing an appropriate constant $C_{1}$. The range from $y^{+}=10$ to 100 is of significant importance. A run list in Table 3 is proposed, according to a comparison of $L^{+}$and $\sigma^{+}$from the RANS channel flow solution, plotted in Fig. 3. The choices of $C_{1}$ are such that $\sigma^{+}$is comparable to $L^{+}$from $y^{+}=10$ to 100 . The other IVM parameters, set to unity, are to be tuned later. The vortex free path follows the approach of the original VM in Eq. (19), with its moving direction changing between successive time steps.

The plots of velocity fluctuations generated at inlet by the IVM with different $C_{1}$ are shown in Fig. 4 , in comparison with the pe- 


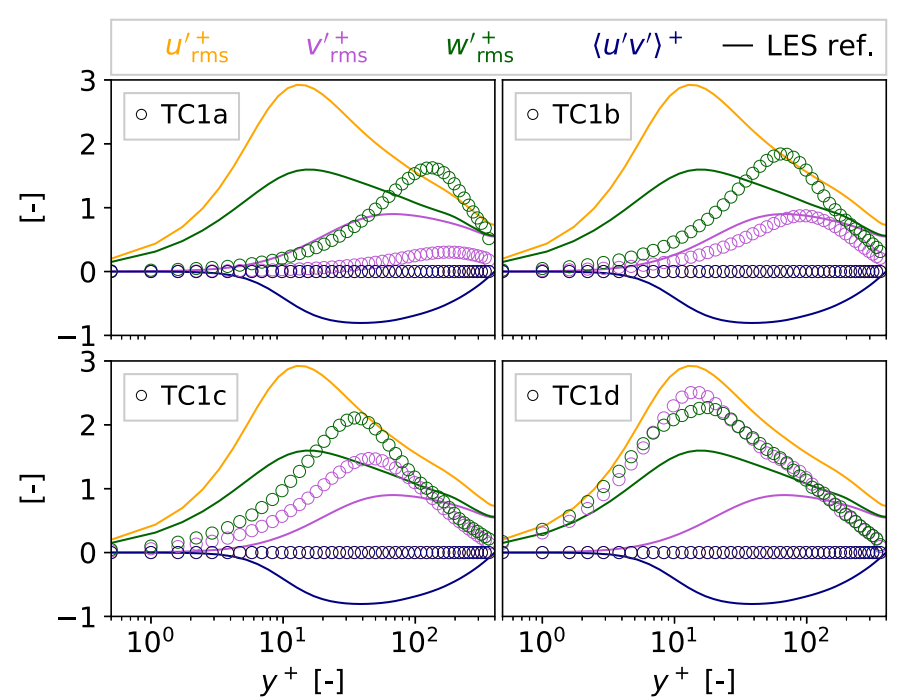

Fig. 4. Channel flow velocity fluctuation profiles for different values of $C_{1}$ at inlet.
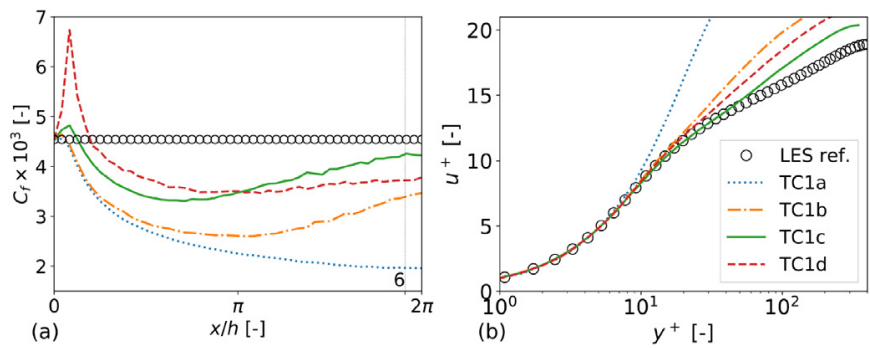

Fig. 5. Channel flow friction coefficient $C_{f}$ (a) and mean velocity profiles at $x=6 \mathrm{~h}$ (b) with different values of $C_{1}$.

riodic channel flow LES. The velocity fluctuations ${u_{\text {rms }}^{+}}^{+}$and the Reynolds shear stress $\left\langle u^{\prime} v^{\prime}\right\rangle^{+}$are null. This is because the IVM is based on a 2D vorticity field, only producing velocity fluctuations within the inlet plane (for the components $v$ and $w$ ). Similar trends are observed for $v_{\text {rms }}^{+}$and $w_{\text {rms }}^{+}$: the smaller the constant $C_{1}$, the higher the peak value and the shorter the wall distance of the peak.

The vortex radius can affect the velocity fluctuations at the inlet, but the most important issue is the streamwise development of turbulence. The tuned coefficient $C_{1}$ should allow the IVM simulation to recover the correct value of $C_{f}$ and the profiles of the periodic LES as soon as possible downstream of the inlet.

The wall friction coefficient $C_{f}$ of the channel flow using the IVM with four different values of $C_{1}$ is plotted in Fig. 5(a). On the same figure, the $C_{f}$ of the periodic LES is plotted, which is a constant and equals the value at the IVM inlet as the mean velocity profile extracted from the periodic LES results are imposed as the IVM inlet condition. The test case 'TC1a', which has the largest vortex radii, shows a continuous drop of $C_{f}$ from the inlet to the outlet. This indicates that the flow is relaminarising. In the test case 'TC1d', where the vortices have their minimum radii, a big jump of $C_{f}$ is observed near the inlet and it then decreases to a constant value. These two cases indicate that neither too big nor too small vortex radii are favourable to generate turbulence. For the other two test cases, a drop is observed within the first half of the channel length and the $C_{f}$ tends to recover its original value. The test case 'TC1c' achieves the best recovery of $C_{f}$.

The profiles of the channel flow mean velocity and velocity fluctuations at $x=6 \mathrm{~h}$ are plotted in Figs. 5(b) and 6, respectively. Again, the test case 'TC1c' obtains the best agreements with the periodic LES results. One should notice that $u_{\text {rms }}^{+}$and $\left\langle u^{\prime} v^{\prime}\right\rangle^{+}$,

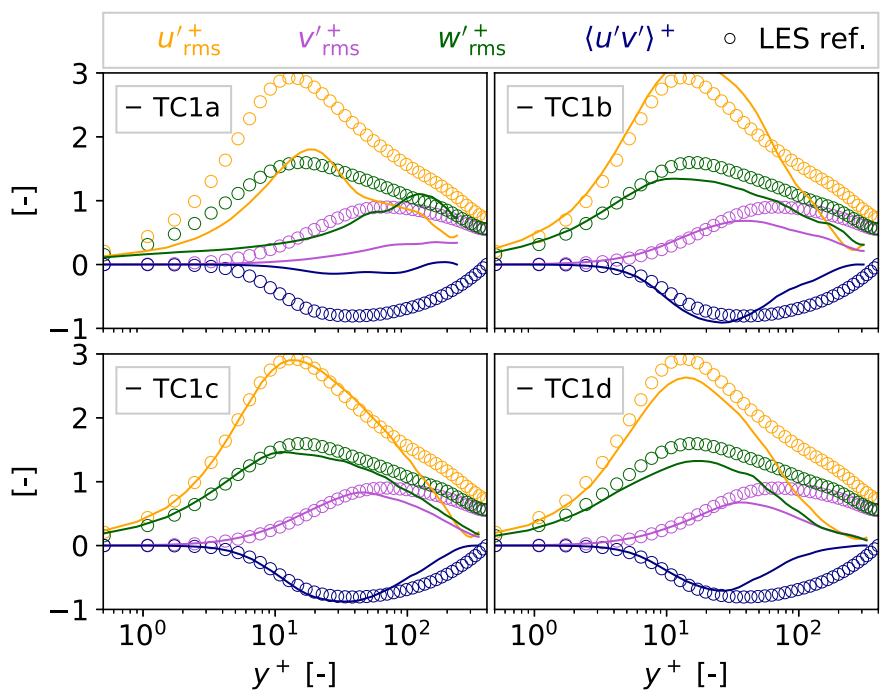

Fig. 6. Channel flow velocity fluctuation profiles at $x=6 h$ with different values of $C_{1}$.

Table 4

Test list for the coefficient tuning of the vortex circulation $\Gamma$.

\begin{tabular}{lllll}
\hline Test case & $C_{1}$ in $\sigma$ & $C_{2}$ in $\Gamma$ & $C_{3}$ in $\lambda$ & $C_{4}$ in $\Delta \tau$ \\
\hline TC1c & $1 / 4$ & 1 & $\lambda=d \lambda$ & 1 \\
TC2a & $1 / 4$ & 1.2 & $\lambda=d \lambda$ & 1 \\
TC2b & $1 / 4$ & 1.6 & $\lambda=d \lambda$ & 1 \\
\hline
\end{tabular}

which are null at inlet due to the absence of streamwise velocity fluctuations, match very well the periodic LES data. This suggests that the 2D velocity fluctuations generated by the IVM are capable to excite the streamwise fluctuating velocity. Therefore 'TC1c', whose radii are well amid the integral length scales as shown in Fig. 3, is selected as the optimal guidance to define the vortex radius $\sigma$.

\section{Vortex circulation $\Gamma$}

The second parameter of the IVM to be tuned is the vortex circulation $\Gamma$. A test list is given in Table 4 . The coefficient $C_{2}$ is calibrated in this part, leaving the vortex free path and reversal time unaltered. Given that the initial guess of $C_{2}=1$ in 'TC1c' refers to the original VM by Sergent, two other larger values are to be tested that may further amplify the velocity fluctuations on the inlet plane. The velocity fluctuations plotted at the inlet with different $C_{2}$ are similar to those in Fig. 4, and are thus omitted for brevity. Thereafter, the inlet velocity fluctuation profiles generated by the IVM during the parameter tuning process will not be plotted. Again, with the IVM, it's not the velocity fluctuation profiles at inlet but the evolution of the flow $\left(C_{f}\right)$ and the characteristics at outlet that are of interest.

The plot of $C_{f}$ with different circulation constants $C_{2}$ is shown in Fig. 7(a). All the three test cases show an evolution of $C_{f}$ decreasing and recovering near its original value, giving satisfactory convergences. Among them, 'TC2a' and 'TC2b' give better recoveries of $C_{f}$ by the end of the computational domain. The mean velocity and velocity fluctuation profiles near the outlet of the channel can be scrutinised in Figs. 7(b) and 8, respectively. The three simulations give good predictions in comparison with the periodic LES results. However, the test cases 'TC2a' and 'TC2b' both show a better agreement with the periodic LES, in terms of the mean velocity profile. This means these two circulation coefficients are both within the reasonable range. To continue the study, the coefficient of 'TC2a' is kept. 

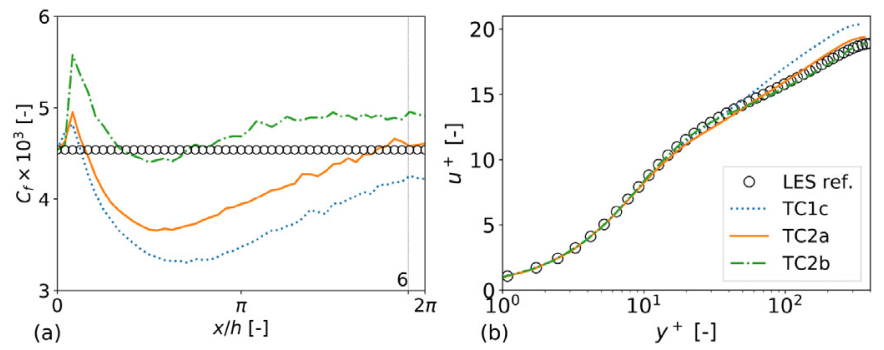

Fig. 7. Channel flow friction coefficient $C_{f}$ (a) and mean velocity profiles at $x=6 \mathrm{~h}$ (b) with different values of $C_{2}$.

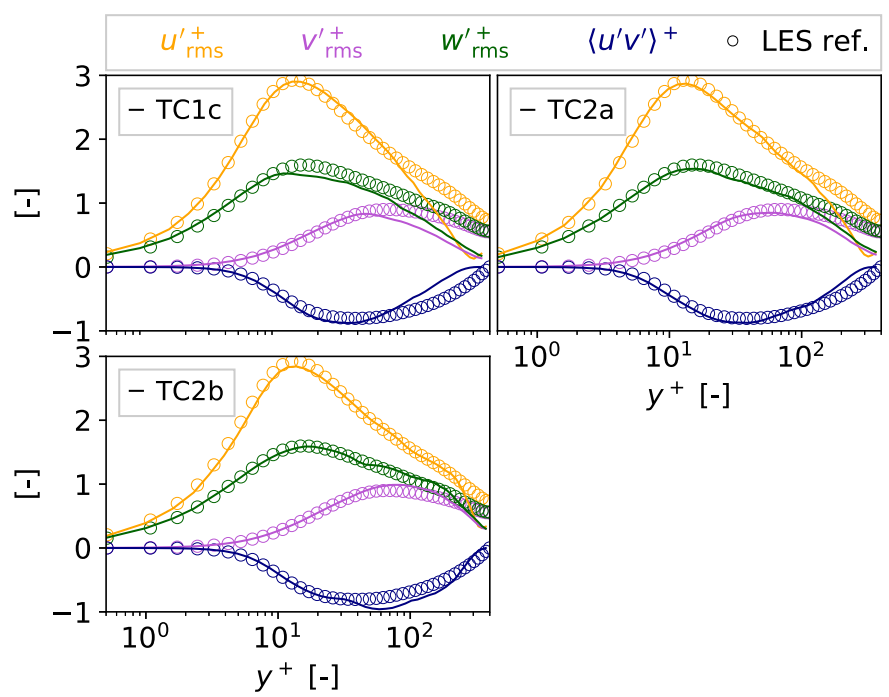

Fig. 8. Channel flow velocity fluctuation profiles at $x=6 h$ with different values of $C_{2}$.

Table 5

Test cases for the coefficient calibration of the vortex free path $\lambda$.

\begin{tabular}{lllll}
\hline Test case & $C_{1}$ in $\sigma$ & $C_{2}$ in $\Gamma$ & $C_{3}$ in $\lambda$ & $C_{4}$ in $\Delta \tau$ \\
\hline TC3a & $1 / 4$ & 1.2 & $1 / 32$ & 1 \\
TC3b & $1 / 4$ & 1.2 & $1 / 16$ & 1 \\
TC3c & $1 / 4$ & 1.2 & $1 / 8$ & 1 \\
\hline
\end{tabular}

Vortex free path $\lambda$

The third IVM parameter to be investigated is the vortex free path $\lambda$. Instead of letting $\lambda=d \lambda$, i.e. vortices change their moving direction between successive iterations, the IVM requests that a vortex must hold its moving direction for a predefined distance $\lambda$. Three test cases are proposed here to tune the third parameter coefficient $C_{3}$, and are summarised in Table 5 . The values are defined from a geometric progression with a common ratio of 2 . The fourth coefficient $C_{4}$ is left to be tested later.

The evolution of the channel flow $C_{f}$ is shown in Fig. 9(a). A common feature is seen for the three cases, i.e. $C_{f}$ encounters a steep drop from the inlet to $2 / 3 \pi$ and then rises gradually towards its original value. A convergence is seen between 'TC3a' and 'TC3b', recovering a $C_{f}$ close to the inlet value. 'TC3c' is different from the former two with a poorer recovery of $C_{f}$, implying that the vortex free path may be excessive. In Figs. 9(b) and 10, the profiles of the mean velocity and velocity fluctuations are plotted in comparison with the periodic channel flow LES. With regard to the mean velocity profile, a very good agreement with the periodic LES is achieved by both 'TC3a' and 'TC3b'. The non-dimensional velocity $u^{+}$is slightly over-predicted by 'TC3c' in the channel centre. A very good agreement is observed for the velocity fluctuations, especially
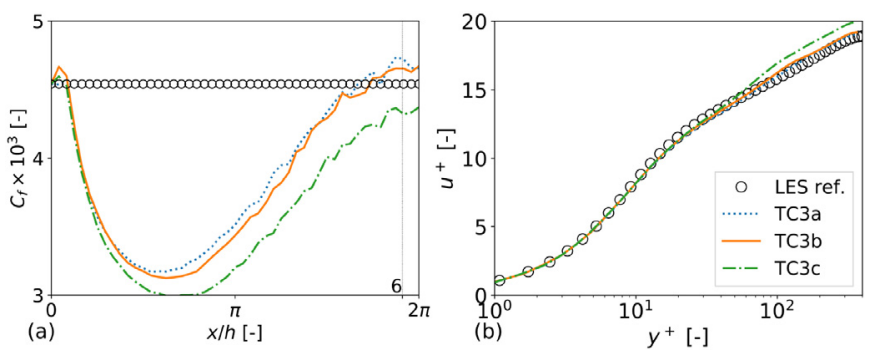

Fig. 9. Channel flow friction coefficient $C_{f}$ (a) and mean velocity profiles at $x=6 \mathrm{~h}$ (b) with different values of $C_{3}$.

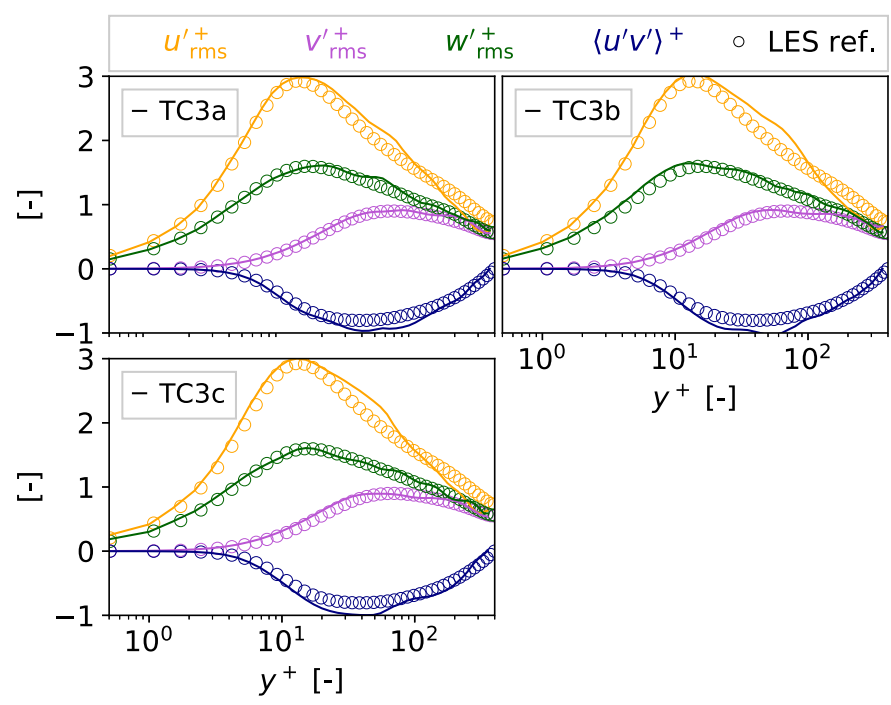

Fig. 10. Channel flow velocity fluctuation profiles at $x=6 h$ with different values of $C_{3}$.

Table 6

Test list for the coefficient tuning of the vortex reversal time

$\Delta \tau$.

\begin{tabular}{lllll}
\hline Test case & $C_{1}$ in $\sigma$ & $C_{2}$ in $\Gamma$ & $C_{3}$ in $\lambda$ & $C_{4}$ in $\Delta \tau$ \\
\hline TC4a & $1 / 4$ & 1.2 & $1 / 16$ & 0.1 \\
TC3b & $1 / 4$ & 1.2 & $1 / 16$ & 1 \\
TC4b & $1 / 4$ & 1.2 & $1 / 16$ & 10 \\
TC4c & $1 / 4$ & 1.2 & $1 / 16$ & 100 \\
\hline
\end{tabular}

in the region far from the wall compared with the previous test cases (Figs. 6 and 8) where the curves approach zero at high $y^{+}$. This suggests that the vortices should be active for a longer period of time to perturb the unsteady flow field, especially for the region far from the wall. To conclude this part, 'TC3c' introduces excessive vortex free path, and a value of $C_{3}$ within the range provided by 'TC3a' and 'TC3b' is reasonable. To continue the study, the coefficient $C_{3}$ is set to the value of 'TC3b' $\left(C_{3}=\frac{1}{16}\right)$.

\section{Vortex reversal time $\Delta \tau$}

The last IVM parameter to be determined is the vortex reversal time $\Delta \tau$, by the end of which a vortex will randomly inverse its rotating direction. A test list is proposed in Table 6 by varying the coefficient $C_{4}$. The option 'TC3b' is the default choice of the original VM.

The development of the channel flow wall friction coefficient $C_{f}$ is shown in Fig. 11(a). 'TC4a', which has the shortest vortex reversal time, relaminarises. The other three cases succeed in reinstating turbulent state. 'TC4b' and 'TC4c' show a converging effect of the vortex reversal time. From the channel flow mean velocity plotted in Figs. 11(b), it is clearly observed that all the cases, except 

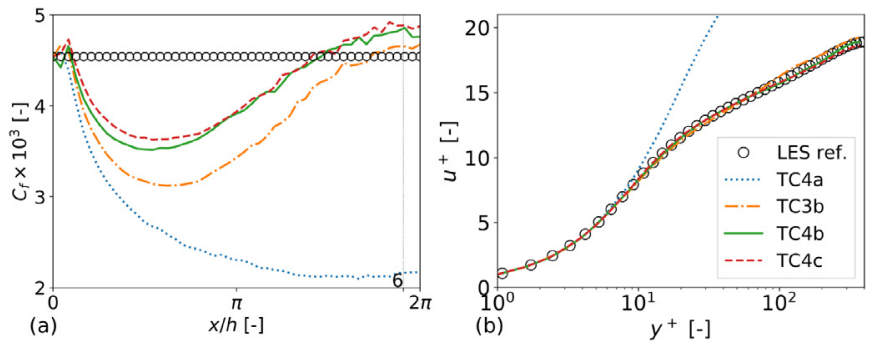

Fig. 11. Channel flow friction coefficient $C_{f}$ (a) and mean velocity profiles at $x=6 \mathrm{~h}$ (b) with different values of $C_{4}$.

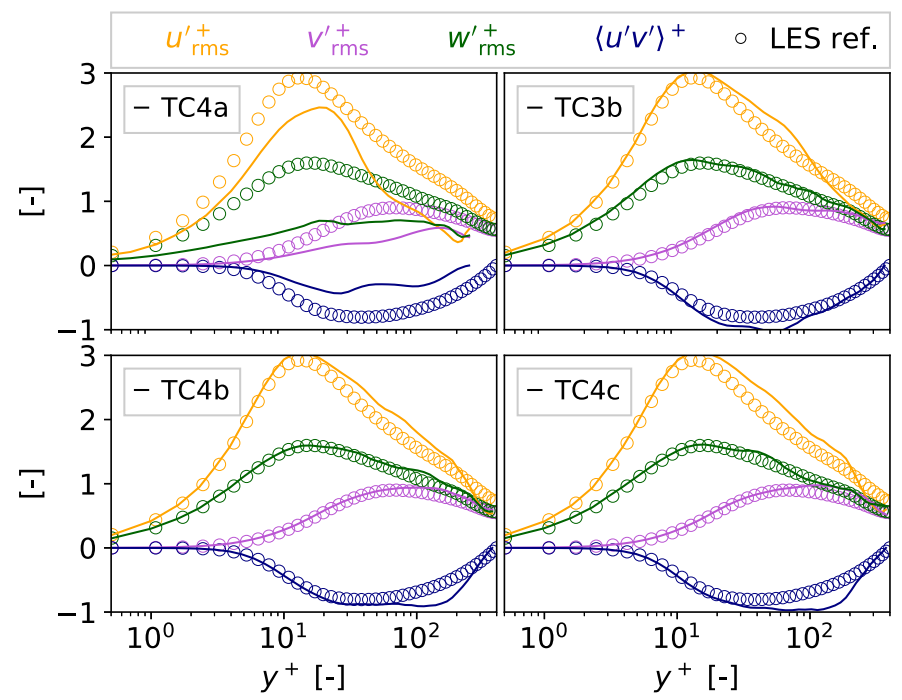

Fig. 12. Channel flow velocity fluctuation profiles at $x=6 h$ with different values of $\mathrm{C}_{4}$.

Table 7

Tuned IVM coefficients for the channel flow at $R e_{\tau}=395$

\begin{tabular}{lllll}
\hline Test case & $C_{1}$ in $\sigma$ & $C_{2}$ in $\Gamma$ & $C_{3}$ in $\lambda$ & $C_{4}$ in $\Delta \tau$ \\
\hline 800 & $1 / 4$ & 1.2 & $1 / 16$ & 10
\end{tabular}

'TC4a', achieve an excellent agreement with the periodic channel flow LES data. A similar observation can be made on the velocity fluctuation profiles, shown in Fig. 12, where the convergence is seen between 'TC4b' and 'TC4c'. Through all the comparisons in this section, 'TC4b' appears to provide a sufficient vortex reversal time.

Till now, the turbulence generated by the IVM has been validated with the periodic channel flow LES results in terms of the wall friction coefficient, the mean velocity profile and the velocity fluctuations. Thus 'TC4b' is selected as the calibration of the IVM for the channel flow at $R e_{\tau}=395$, with the best choices of all the four IVM coefficients, which are listed in Fig. 7.

\section{Convergence check with longer channel: $3 \pi \mathrm{h}$}

The channel flow LES with the IVM is further extended to $3 \pi \mathrm{h}$ in the streamwise direction to evaluate the convergence of the adaptive distance. The comparison of the friction coefficient $C_{f}$ is in Fig. 13(a) between the $2 \pi h$ and the $3 \pi h$ channels. The convergence of $C_{f}$, which represents the convergence of the mean flow, is achieved at about $x=6 h$ in both cases.

A further scrutinisation is made to check the balance of energy transfer of the turbulence generated. As proposed in this paper, this can be measured by the velocity-derivative skewness $S_{k}$. The evolution of the $S_{k}$ of the turbulent channel flow with the opti-
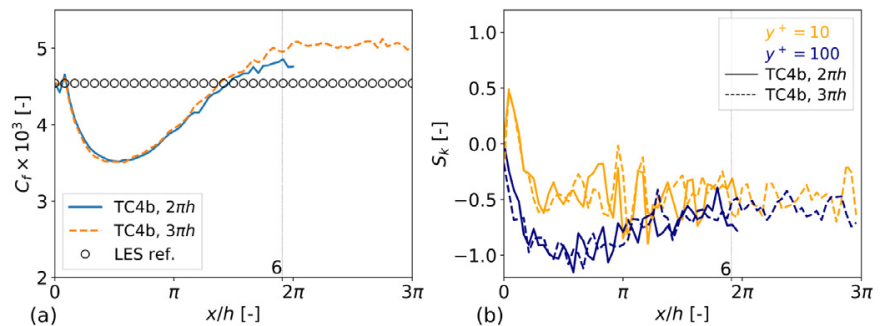

Fig. 13. Channel flow results with different channel lengths: $2 \pi h$ vs $3 \pi h$. (a) Friction coefficient $C_{f}$, (b) Velocity-derivative skewness $S_{k}$.
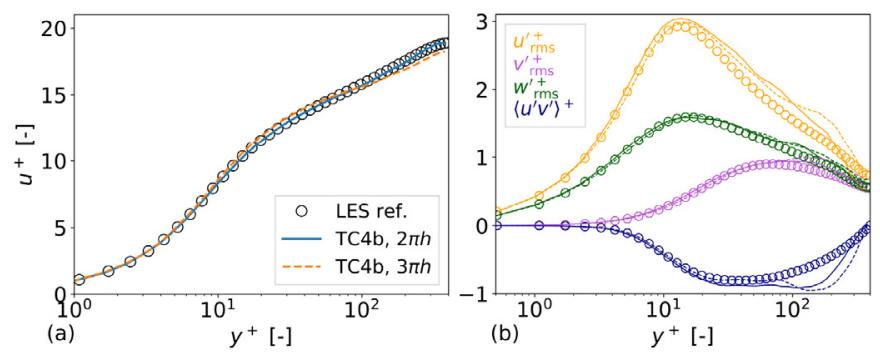

Fig. 14. Mean velocity (a) and velocity fluctuation (b) profiles in different channel lengths: $2 \pi h$ vs $3 \pi h$.

mised IVM is plotted at $y^{+}=10$ and 100 in Fig. 13(b) for both the $2 \pi h$ and the $3 \pi h$ channels. For both curves of the $2 \pi h$ channel, $S_{k}$ is null at inlet as no streamwise velocity fluctuation is given by the IVM. Downstream of the inlet, $S_{k}$ evolves until $x / h=0.5 \pi$. The oscillations then gradually stabilise around $S_{k}=-0.5$ at $x / h=6$, indicating that high quality turbulence is established, and the energy transfer is balanced between large- and small-scale motions. Note that the value of $S_{k}=-0.5$ is commonly used as reference, in various two-point studies of turbulence [38]. A comparable feature is observed between the evolutions of $C_{f}$ and $S_{k}$ : both evolve downstream of the IVM inlet and stabilise around a constant value close to the outlet. This means both the mean velocity field (measured by $C_{f}$ ) and energy equilibriums (evaluated by $S_{k}$ ) comply with real turbulence. These two evolutionary parameters should be considered together when evaluating a turbulent flow generated by the IVM. A very similar behaviour is observed on the curves of the $3 \pi h$ channel: $C_{f}$ and $S_{k}$ are already converged at $x=6 h$ and are not altered by the increase of the channel length. The comparisons of the mean velocity profiles and the Reynolds stresses are shown in Fig. 14 between the $2 \pi h$ and the $3 \pi h$ channels. The profiles of the $2 \pi h$ channel are extracted at $x=6 h$, while those of the $3 \pi h$ channel are obtained at $x=9.3 h$. The overlapping profiles shown in the figure reveal that an adaptive distance of $6 h$ is long enough for a channel flow LES with the IVM to be statistically converged. This is consistent with the evolutions of $C_{f}$ and $S_{k}$. Compared with the original VM that requires $x / h=12$ [39] and the synthetic-eddy method of Jarrin et al. [17] that requires at least $x / h=10$ to achieve a fully developed turbulent channel flow, the IVM is very efficient, in terms of adaptive distance $(x / h=6)$ to obtain high quality turbulence.

\subsubsection{Channel flow LES with IVM at $R e_{\tau}=590$ : validation}

The IVM is further validated with a channel flow at a higher turbulent Reynolds number $R e_{\tau}=590$ [36]. The dimensions of the channel are again $2 \pi h \times 2 h \times \pi h$ in the streamwise, wall-normal and spanwise directions, respectively. $h=0.01 \mathrm{~m}$ is the channel half height. With regard to the mesh, $73 \times 131 \times 61$ points are allocated using uniform grids in the $x$ and $z$ directions and tanh distribution in the wall normal direction. This yields grid resolutions in wall units: $\Delta x^{+}=52, \Delta y^{+}=0.5$ and $\Delta z^{+}=31$. The same bound- 

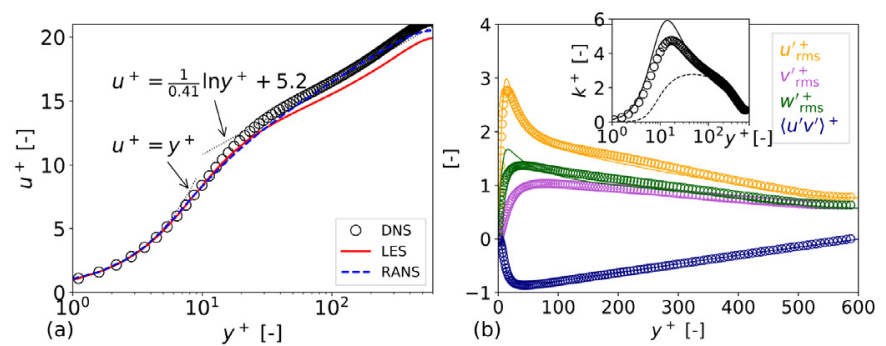

Fig. 15. Channel flow mean velocity (a) and velocity fluctuation (b) profiles, at $R e_{\tau}=590$. DNS data from Kim et al. [36].
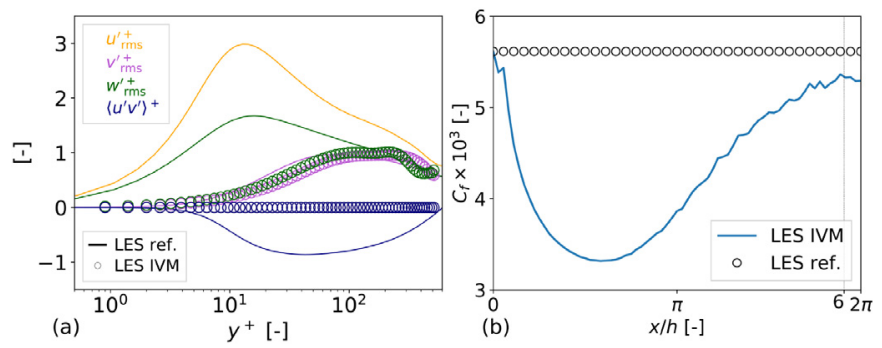

Fig. 16. Channel flow $\left(R e_{\tau}=590\right)$ velocity fluctuation profiles at inlet (a) and friction coefficient $C_{f}$ (b).

ary conditions are employed as for the channel flow LES with the IVM at $R e_{\tau}=395$. The mean flow and turbulent variables of the inflow are initialised by RANS results at $R e_{\tau}=590$. The time step used is $5 \times 10^{-8} \mathrm{~s}$, corresponding to a Courant number of 2.1 . Numerical tests revealed that the IVM coefficients tuned on the $R e_{\tau}=395$ channel test case, listed in Table 7, are well adapted to this $R e_{\tau}=590$ case. This is illustrated as follows.

Prior to the IVM simulation, a periodic LES with streamwise periodic boundary conditions have been conducted and compared with the DNS of Kim et al. [36] and RANS. The comparisons of mean velocity and velocity fluctuation profiles are shown in Fig. 15. A good agreement of the mean velocity profiles is observed. A slight shift from the DNS is found by LES in the log layer and is considered to be due to the slight overestimate of $u_{\tau}$. With regard to the velocity fluctuations, the inner peak is well captured by the LES with a slight over-prediction of the peak amplitude. The RANS turbulent kinetic energy profile is compared with those of the DNS of Kim et al. [36] and the LES, as RANS models based on Boussinesq assumption cannot represent the anisotropy of the Reynolds stresses. It should be noted that the standard $k-\omega$ model doesn't correctly capture the peak of $k$. The influence of this flaw on the IVM turbulence generation will be investigated. The velocity fluctuations generated by the IVM on the 2D inlet plane are illustrated in Fig. 16(a). The two components $v_{\text {rms }}^{+}$and $w_{\text {rms }}^{\prime+}$ are comparable to the $v_{\text {rms }}^{+}$profile of the periodic channel flow LES, while the other two components are null as they involve $u^{\prime}$ which is not produced by the IVM on the inlet plane.

The friction coefficient $C_{f}$, plotted in Fig. 16(b), initiates at $5.5 \times$ $10^{-3}$, then decreases steeply, and progressively recovers near its initial value at about $x=6 h$.

The profiles of the channel flow mean velocity and velocity fluctuations are extracted near the outlet at $x=6 h$ and shown in Fig. 17. The mean velocity profile $u^{+}$of the IVM achieves a very good agreement with the periodic channel flow data. About the velocity fluctuations, very good agreements are observed for the terms $v_{\text {rms }}^{\prime+}, w_{\text {rms }}^{\prime+}$ and $\left\langle u^{\prime} v^{\prime}\right\rangle^{+}$, whilst the comparison of the velocity fluctuations $u_{\text {rms }}^{+}$is acceptable between the IVM and periodic LES data, showing a gentler drop above the peak in the IVM. It should be reminded that the same IVM coefficients, tuned in the
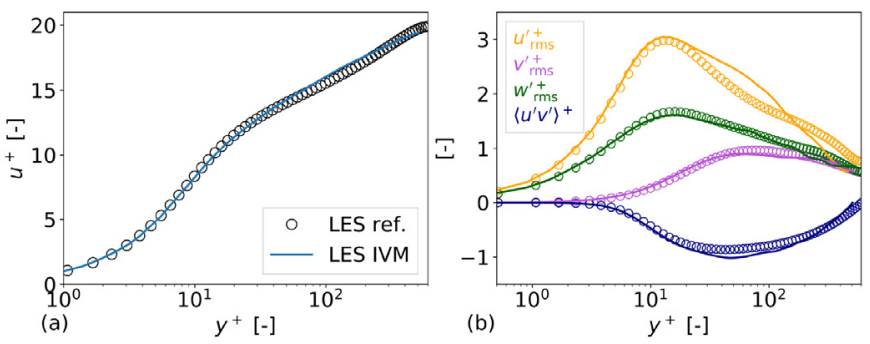

Fig. 17. Profiles of the channel flow $\left(R e_{\tau}=590\right)$ mean velocity (a) and velocity fluctuations (b) at $x=6 h$.

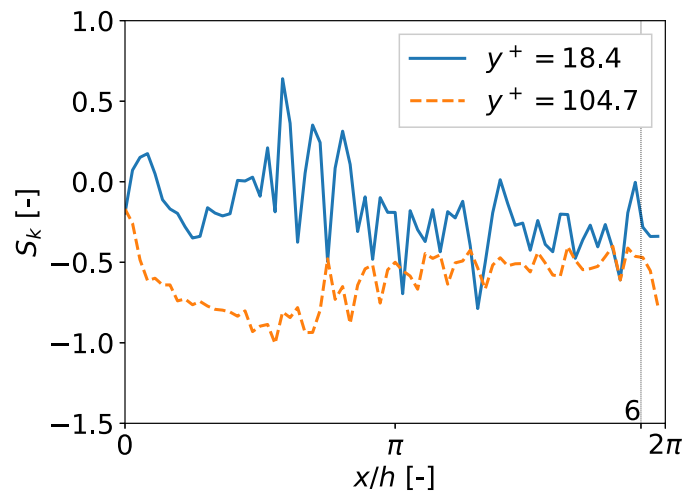

Fig. 18. Velocity-derivative skewness $S_{k}$ for the channel flow $\left(R e_{\tau}=590\right)$ LES with IVM.

channel flow at $R e_{\tau}=395$, are directly applied to the channel flow at $R e_{\tau}=590$ in this section. This indicates some robustness of the IVM.

The evolutions of the channel flow velocity-derivative skewness $S_{k}$ at two near-wall positions are plotted in Fig. 18. They both have an initial value around 0 and stabilise by oscillating around a constant value of -0.5 . This indicates that realistic turbulence is established, in which the transfers of energy between different scales are balanced.

To summarise this section, synthetic velocity fluctuations have been generated by the IVM at the inlet of a channel flow at $R e_{\tau}=$ 590, and high-quality turbulence established within a short adaptive distance $x / h=6$.

\subsection{Flat-plate boundary layer}

Generating turbulent boundary layers is particularly useful as they are usually required as inflow condition for the main computation downstream, as mentioned in Introduction. A turbulent boundary layer can be initialised from a laminar boundary layer at the inlet and forced to transition at sufficiently high Reynolds number. Fully developed turbulence will be established after the transition. This process often takes a long streamwise extent. The idea is to reduce the computing costs by setting the IVM on a plane where the flow state is fully developed, upstream of the position of the desired turbulent Reynolds number. In the present section, a classic flat-plate boundary layer LES is conducted and is used as reference to validate the flat-plate boundary layer LES with the IVM.

\subsubsection{Full-length flat-plate boundary layer LES at $\operatorname{Re}_{\delta_{2}}=1968$ (reference LES)}

The IVM is tested with a flat-plate boundary layer at $R e_{\delta_{2}}=$ 1968, inspired by Jiménez et al. [40]. The reference geometry defined in [39] is used in this study: the streamwise extent $L_{x}=$ $0.3 \mathrm{~m}$, the wall-normal height $L_{y}=2 \delta\left(x=L_{x}\right)=11.7 \mathrm{~mm}$ and the 

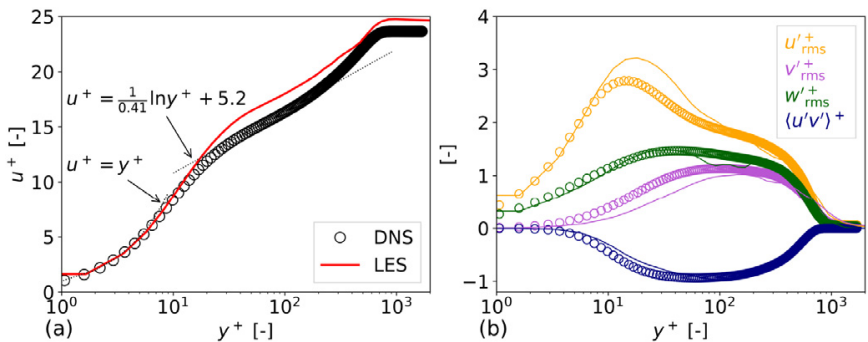

Fig. 19. Mean velocity (a) and velocity fluctuation (b) profiles of the full-length flatplate boundary layer at $R e_{\delta_{2}}=1968$.

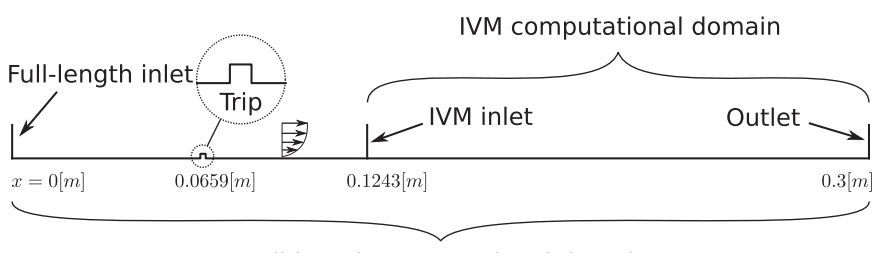

Full-length computational domain

Fig. 20. Sketch of the IVM implementation in the flat-plate boundary layer simulation.

spanwise width $L_{z}=\delta\left(x=L_{x}\right)=5.86 \mathrm{~mm}$, where $\delta$ is the nominal boundary layer thickness estimated by the $1 / 7$ power-law velocityprofile model [22]. The spanwise boundaries are connected by a periodic boundary condition, and the upper boundary with $-0.5^{\circ}$ inclination is set as outlet. The mesh of the computational domain consists of $829 \times 39 \times 43$ points, yielding grid resolutions of $\Delta x^{+}<83, \Delta y^{+}<2$ and $\Delta z^{+}<32$. A global constant time step of $4 \times 10^{-8} \mathrm{~s}$ is used for the flat-plate LES simulations, which gives a Courant number of 1.8 . The laminar-turbulent transition is forced by a source term that acts as a tripping band, following Boudet et al. [39].

A comparison of the LES results with the DNS data of Jiménez et al. for the full-length flat-plate boundary layer [40] is shown in Fig. 19. Reasonably good agreements are observed between the LES and DNS. A slight difference in the mean velocity profiles appears to be due to the underestimate of the friction velocity $u_{\tau}$ by the LES. Hereafter, these full-length LES results are used as reference to tune and validate the IVM.

\subsubsection{Flat-plate boundary layer LES with IVM}

With the IVM, computing costs can be reduced by simulating only a downstream proportion of the flat-plate boundary layer. A sketch is presented in Fig. 20. The IVM computational domain starts from a plane after the transition, at $\operatorname{Re}_{\delta_{2}}=988(x=$ $0.1243 \mathrm{~m}$ ), on which the IVM is implemented, and terminates at the same position as the full-length LES. The profiles of mean velocity, turbulent kinetic energy and turbulent dissipation rate, which are required by the IVM, are calculated from the reference LES at $R e_{\delta_{2}}=988$. Note that the IVM inlet could be also initialised with RANS solutions as discussed above. But in this section, the RANS simulation is omitted since the available full-length LES data can provide information for the IVM initialisation, and RANS simulations would give good statistics similar to the LES for this kind of simple-geometry flows. Since the flow features of a flat-plate boundary layer are different from a channel flow, the IVM parameters need to be re-tuned. For brevity, only the tuned constants are presented in this section, as the procedure of tuning has been introduced previously. The coefficients are listed in Table 8. In this test case, 400 vortices are injected on the inlet plane. The coefficient of the vortex radius is set to $C_{1}=1 / 80$, according to the integral length scale at $R e_{\delta_{2}}=988$ plotted in Fig. 21(a). The circulation of the vortices is decreased $\left(C_{2}=0.5\right)$ to avoid excessive fluctua-
Table 8

Tuned IVM coefficients for the flat-plate boundary layer at $R e_{\delta_{2}}=1968$.

\begin{tabular}{lllll}
\hline Test case & $C_{1}$ in $\sigma$ & $C_{2}$ in $\Gamma$ & $C_{3}$ in $\lambda$ & $C_{4}$ in $\Delta \tau$ \\
\hline 400 & $1 / 80$ & 0.5 & $1 / 16$ & 10 \\
\hline
\end{tabular}
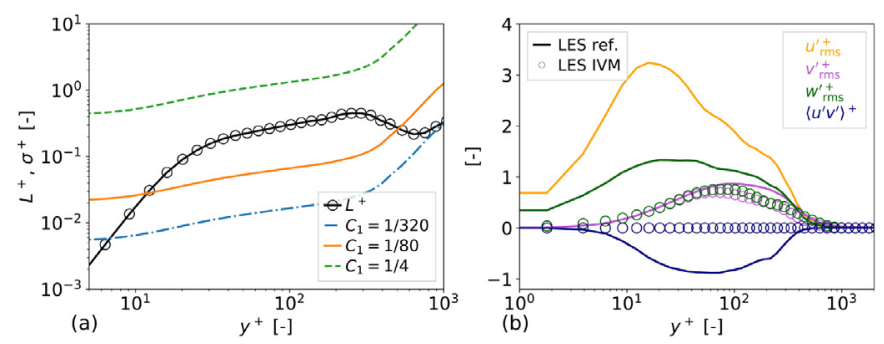

Fig. 21. (a) Comparison of $\mathrm{L}^{+}$and $\sigma^{+}$obtained from the flat-plate boundary layer LES solution at $R e_{\delta_{2}}=988$. (b) Velocity fluctuation profiles at inlet for the flat plate IVM LES, at $R e_{\delta_{2}}=988$.

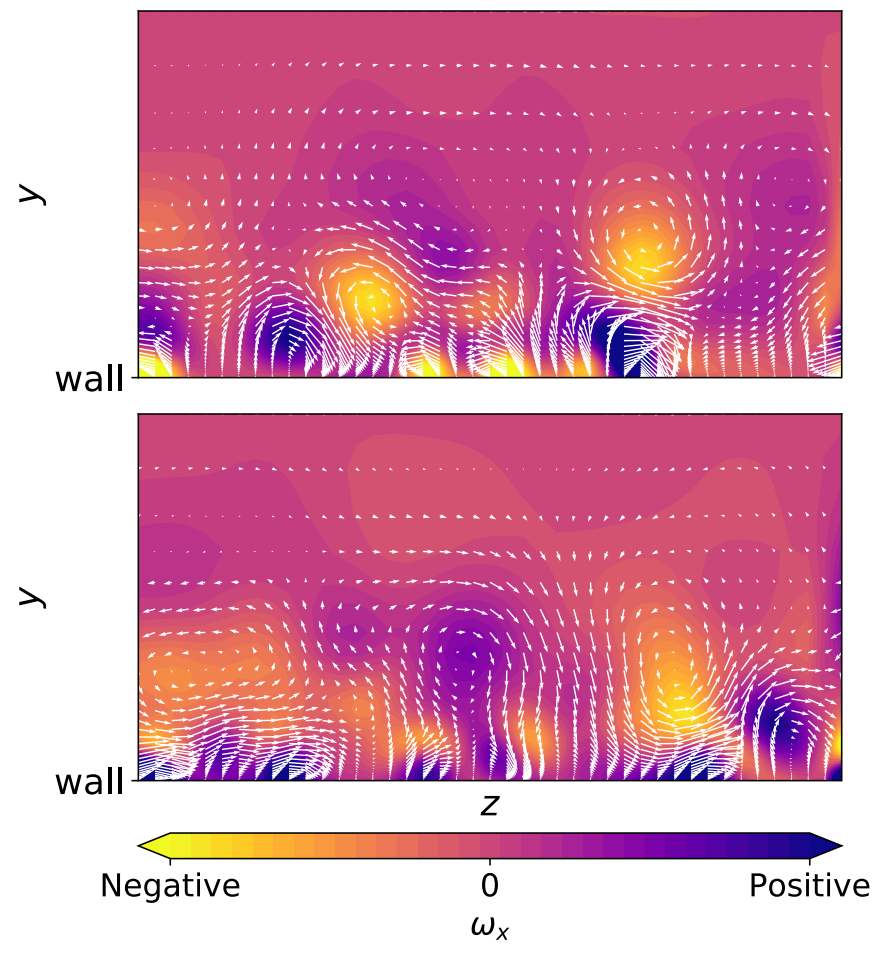

Fig. 22. Instantaneous streamwise vorticity contours with velocity vectors on the inlet plane, at two different iterations, for the flat plate IVM LES.

tions, as the size of the vortices is smaller than that of the channel flow. The other two parameters are found insensitive to the changes from the channel flows to the flat-plate boundary layer.

The velocity fluctuations generated by the IVM on the inlet plane are plotted in Fig. 21(b). The components ${u_{\text {rms }}^{+}}^{+}$and $\left\langle u^{\prime} v^{\prime}\right\rangle^{+}$ are null as no fluctuation is excited in streamwise direction by the IVM. $v_{\text {rms }}^{+}$and $w_{\text {rms }}^{+}$generated by the IVM have the same order of magnitude as those of the reference LES but are closer to each other. Snapshots of the instantaneous streamwise vorticity along with velocity vectors built by the IVM on the inlet plane are shown in Fig. 22. Vorticity contours with positive and negative values are combined by the vortices of different radii and circulations injected on the inlet plane. Differences between the two snapshots are obvious, suggesting that the vortices are indeed enough active to move away from their original positions and to inverse their rotating directions. The vortices are very active in the near-wall re- 

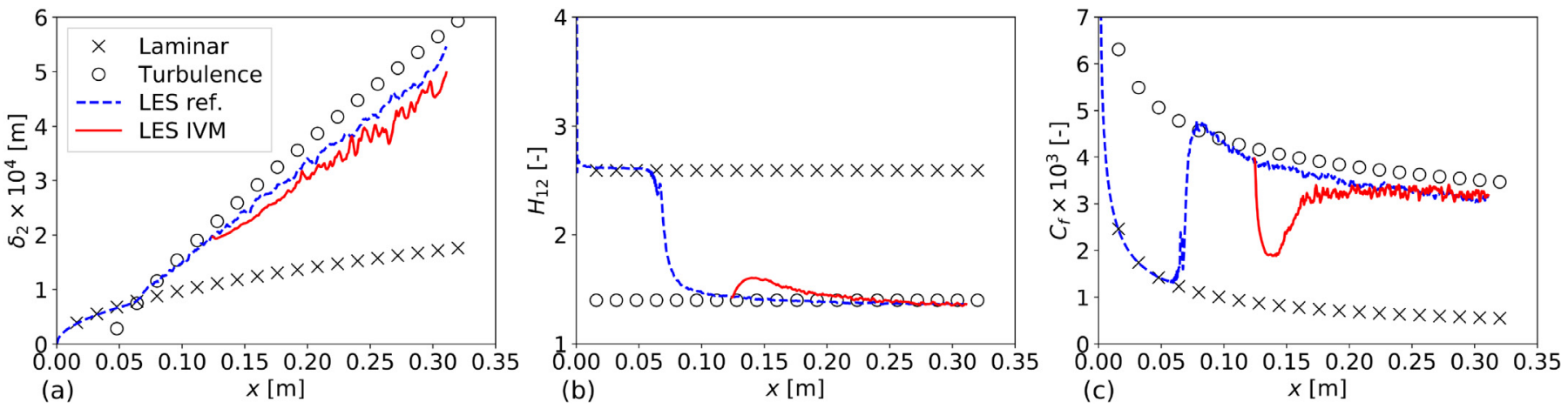

Fig. 23. Evolution of the flat-plate boundary layer: comparison between the reference LES and IVM LES. (a) $\delta_{2}$, (b) $H_{12}$, (c) $C_{f}$.

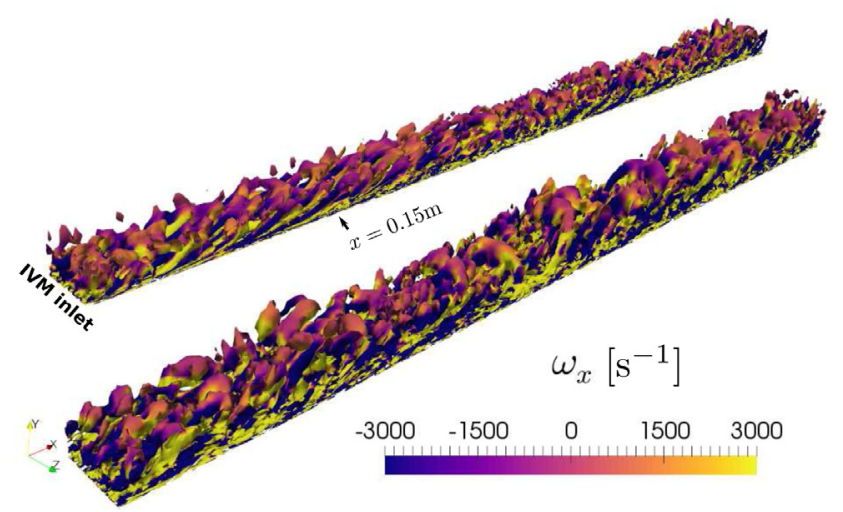

Fig. 24. Coherent structures revealed by the $Q$-criterion $\left(Q=2 \times 10^{5}\left[\mathrm{~s}^{-2}\right]\right)$, coloured by streamwise vorticity $\omega_{x}$, for the flat plate IVM LES. For visualisation, the total length is split into two segments, staggered from top to bottom.

gion where the turbulent behaviour is intense. Also, the vanishing amplitude of the velocity vectors on the wall is consistent with the no-slip wall treatment. The IVM provides initial 2-D perturbations at inlet, but the investigation must analyse how turbulence develops downstream. Fig. 23presents a comparison of the flat-plate boundary layer evolution between the reference LES and the IVM LES. The IVM LES starts at $\operatorname{Re}_{\delta_{2}}=988(x=0.1243 \mathrm{~m})$. The evolution of the momentum thickness $\delta_{2}$ is plotted in Fig. 23(a), in comparison with Blasius' solution (laminar) and Michel's law (turbulent). The curve of the IVM LES starts at the same $\delta_{2}$ as the reference LES (used for initialisation) and follows the $\delta_{2}$ development of the reference LES data with a slight under-prediction. The shape factor $\mathrm{H}_{12}$ is illustrated in Fig. 23(b). A tendency of relaminarisation is seen at $x \approx 0.15 \mathrm{~m}$, but $H_{12}$ re-approaches soon the classic turbulent value. A similar relaminarisation trend is observed in the evolution of the friction coefficient $C_{f}$, depicted in Fig. 23(c), before reapproaching the reference LES at about $x=0.175 \mathrm{~m}$. The instantaneous turbulent coherent structures are revealed by the Q-criterion in Fig. 24. The short transition process is observed at $x \approx 0.15 \mathrm{~m}$ with large-scale structures that transition into smaller structures downstream. These results indicate that the velocity fluctuations generated by the IVM allow the flow to recover the turbulent behaviour.

The mean velocity and velocity fluctuation profiles at $R e_{\delta_{2}}=$ 1968 obtained with the IVM are compared with those from the reference LES, in Fig. 25. All the profiles of the IVM LES are in good agreement with the ones of the reference data. This further evidences that the velocity fluctuations generated by the IVM allow a quick establishment of turbulence.
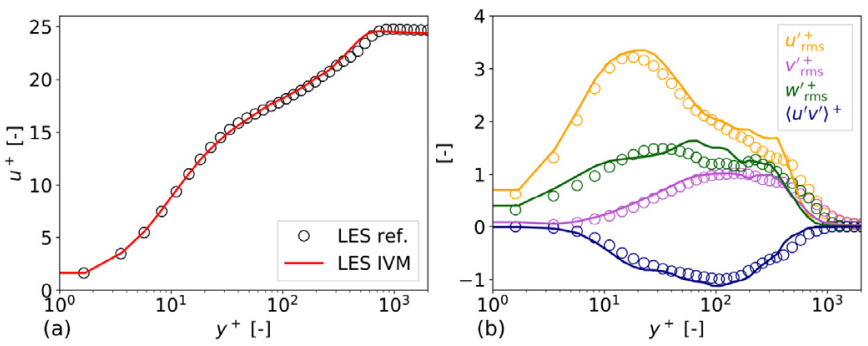

Fig. 25. Profiles of the flat-plate boundary layer mean velocity (a) and velocity fluctuations (b) at $R e_{\delta_{2}}=1968$.

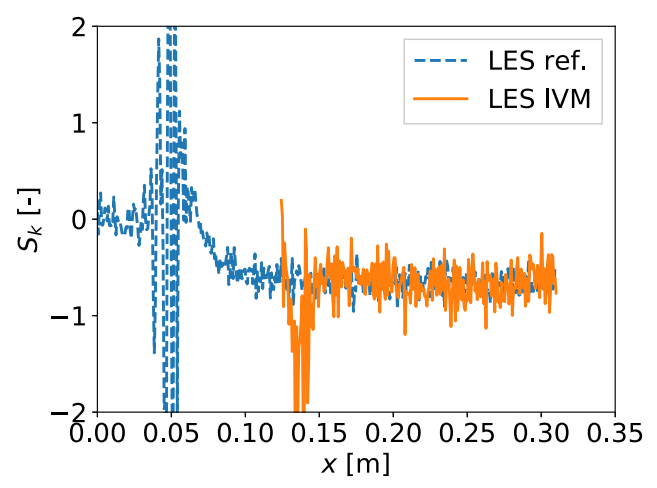

Fig. 26. Evolution of the flat-plate boundary layer velocity-derivative skewness $S_{k}$ at $y^{+}=26.7$ and $\operatorname{Re}_{\delta_{2}}=1968$.

In addition to the previous conventional analyses, the velocityderivative skewness $S_{k}$, which relates to the balance of energy transfer, is scrutinised in Fig. 26, at a near-wall location corresponding to $y^{+}=26.7$ for $R e_{\delta_{2}}=1968$. In the reference full-length flat-plate LES, a uniform velocity is imposed at inlet and the flow is transitioned by a tripping band at $x \approx 0.05 \mathrm{~m}$. $S_{k}$ is around 0 downstream of the inlet and finally oscillates slightly about a constant value of -0.7 after a significant variation caused by the transition. Concerning the IVM LES, $S_{k}$ initiates at 0 as the IVM does not provide fluctuations in the streamwise direction on the inlet plane. After a short transient process, which is also visible in the evolutions of $H_{12}$ and $C_{f}$, the IVM LES and the reference LES overlap each other. This confirms that the turbulence of both simulations achieves high quality, in terms of the balance of energy transfer between large- and small-scale fluid motions. In particular, the IVM provides a very efficient way of developing high-quality turbulence, in terms of adaptive distance. In agreement with the evolutions of $H_{12}$ and $C_{f}$, it appears that high-quality turbulence with equilibrium of energy transfer between different scale motions is established at $x=0.175 \mathrm{~m}$, corresponding to an adaptive 
distance of $21 \delta(x=0.175 m)$. This value appears large compared with the adaptive distance for channel flows. However, the turbulent Reynolds number is greater than for the channel flow cases, and this might reduce the efficiency of the synthetic turbulence methods, as suggested by Jarrin et al. [17].

\section{Conclusions}

The present paper analysed and improved the VM of Sergent [1] for generating LES inflow conditions, which are one of the most important issues in performing LES for industrial applications. Numerical tests, in channel and flat-plate flows, have been conducted. In all the cases, with the aim of providing reference data, classic LES were conducted and validated against DNS. Using the same flow configurations, results of spatially developing LES with the IVM were checked against these reference data. In order to better assess the results of LES, we also introduced a new criterion based on velocity-derivative skewness, which evaluates the balance of energy transfer between large- and small-scale fluid motions.

The five parameters involved in the IVM are :

1. vortex number $N$. The effect of the number of vortices has been investigated by Sergent [1], and 800 and 400 have been used respectively for the channel flow and the flat-plate boundary layer in this paper.

2 . vortex radius $\sigma$. It should be comparable to the integral length scale from the point of view of the energy cascade, where large-scale eddies are responsible for the containing and transferring of energy.

3. vortex circulation $\Gamma$. It is determined by the root mean square of the local velocity fluctuation derived from the local turbulent kinetic energy assuming isotropic turbulence.

4 . vortex free path $\lambda$. This is the distance travelled by a vortex between two successive changes in its moving direction. $\lambda$ should have an order of magnitude similar to the vortex radius $\sigma$.

5 . vortex reversal time $\Delta \tau$. The vortex reversal time is based on the turbulence time scale.

For IVM simulations, the evolution of turbulence and its downstream characteristics are of great importance, and depend on the IVM parameters. Parameter calibrations and their validations on academic channel flows have demonstrated that the IVM can significantly reduce the adaptive distance. The friction coefficient, the mean velocity and the velocity fluctuation profiles can achieve excellent agreements with the reference data, at an adaptive distance of only $6 h$ for channel flows, downstream of the IVM inlet. This constitutes a significant improvement, in terms of adaptive distance, compared with the VM of Sergent $[1,15]$ (12 h) and the synthetic eddy method of Jarrin et al. [17,18] (10h). The application on a flat-plate boundary layer has also proved the capability and efficiency (measured again in terms of adaptive distance) of the IVM. The adaptive distance to establish turbulence for the flat-plate boundary layer studied is $21 \delta$ ( $\delta$ is the target nominal boundary layer thickness).

The success of the IVM in the test cases demonstrates its applicability for both internal and external flows. The establishment of turbulence is faster compared with other methods. The IVM, therefore, has a great potential to be applied to more complex geometries.

In addition to the conventional criteria, the evolution of the velocity-derivative skewness has been scrutinised to assess the development of turbulence. Similarly to what is usually observed in experiments of grid turbulence and DNS, downstream of the inlet, calculations with the inflow conditions generated by the IVM show that the development of turbulence is closely linked with the velocity-derivative skewness $S_{k}$. Within the adaptive region, the value of $S_{k}$ originates from 0 , then decreases and increases back to a certain negative level. Finally, by the end of the adaptive distance, it stabilizes around a negative value about -0.5 in the fully developed turbulence region. It indicates that the energy transfer between different scales is balanced with the turbulent dissipation. Meanwhile, it is observed that the friction coefficient $C_{f}$ behaves in a similar way and recovers to the expected value. Once the convergences of $C_{f}$ and $S_{k}$ are achieved, the turbulent flow reaches its fully developed state, i.e. the mean flow and the Reynolds stresses converge. Thus we propose to use the velocity-derivative skewness as a new criterion to assess LES results.

\section{Acknowledgements}

Baolin Xie was financially supported by the China Scholarship Council during his Ph.D. at École Centrale de Lyon.

\section{References}

[1] Sergent E. Vers une mthodologie de couplage entre la simulation des grandes chelles et les modles statistiques. Ecole centrale de Lyon; 2002.

[2] Duchaine F, Dombard J, Gicquel LYM, Koupper C. On the importance of inlet boundary conditions for aerothermal predictions of turbine stages with large eddy simulation. Comput Fluids 2017;154:60-73. doi:10.1016/j.compfluid.2017. 05.024.

[3] Gao F, Ma W, Zambonini G, Boudet J, Ottavy X, Lu L, Shao L. Large-eddy simulation of 3-D corner separation in a linear compressor cascade. Phys Fluids 2015;27(8):085105. doi:10.1063/1.4928246.

[4] Boudet J, Cahuzac A, Kausche P, Jacob MC. Zonal large-eddy simulation of a fan tip-clearance flow, with evidence of vortex wandering. J Turbomach 2015;137(6):061001-061001-9. doi:10.1115/1.4028668.

[5] Boudet J, Caro J, Li B, Jondeau E, Jacob MC. Zonal large-eddy simulation of a tip leakage flow. Int J Aeroacoustics 2016;15(6-7):646-61. doi:10.1177/ $1475472 X 16659215$

[6] Keating A, Piomelli U, Balaras E, Kaltenbach HJ. A priori and a posteriori tests of inflow conditions for large-eddy simulation. Phys Fluids (1994-present) 2004;16(12):4696-712.

[7] Tabor GR, Baba-Ahmadi MH. Inlet conditions for large eddy simulation: a review. Comput Fluids 2010;39(4):553-67.

[8] Sagaut P, Deck S, Terracol M. Multiscale and multiresolution approaches in turbulence: LES, DES and hybrid RANS/LES methods: applications and guidelines. World Scientific; 2013.

[9] Lund TS, Wu X, Squires KD. Generation of turbulent inflow data for spatially-developing boundary layer simulations. J Comput Phys 1998;140(2):233-58.

[10] Rosenhead L. The formation of vortices from a surface of discontinuity. Proc $R$ Soc London Series A 1931;134(823):170-92.

[11] Maull DJ. An introduction to the discrete vortex method. NASA STI/Recon Technical Report A 1980;81:27614.

[12] Leonard A. Vortex methods for flow simulation. J Comput Phys 1980;37(3):289-335.

[13] Mathey F, Cokljat D, Bertoglio JP, Sergent E. Assessment of the vortex method for large eddy simulation inlet conditions. Prog Comput Fluid Dyn, Int J 2006;6(1-3):58-67. doi:10.1504/PCFD.2006.009483.

[14] Mathey F. Aerodynamic noise simulation of the flow past an airfoil trailing-edge using a hybrid zonal RANS-LES. Comput Fluids 2008;37(7):836-43.

[15] Benhamadouche S, Jarrin N, Addad Y, Laurence D. Synthetic turbulent inflow conditions based on a vortex method for large-eddy simulation. Prog Comput Fluid Dyn, Int J 2006:6(1-3):50-7.

[16] Jarrin N, Benhamadouche S, Laurence D, Prosser R. A synthetic-eddy-method for generating inflow conditions for large-eddy simulations. Int J Heat Fluid Flow 2006;27(4):585-93.

[17] Jarrin N, Prosser R, Uribe JC, Benhamadouche S, Laurence D. Reconstruction of turbulent fluctuations for hybrid RANS/LES simulations using a synthetic-eddy method. Int J Heat Fluid Flow 2009;30(3):435-42.

[18] Poletto R, Craft T, Revell A. A new divergence free synthetic eddy method for the reproduction of inlet flow conditions for LES. Flow Turbul Combust 2013;91(3):519-39.

[19] Pamiès M, Weiss PE, Garnier E, Deck S, Sagaut P. Generation of synthetic turbulent inflow data for large eddy simulation of spatially evolving wall-bounded flows. Phys Fluids (1994-present) 2009;21(4):045103.

[20] Xie B. Improved vortex method for LES inflow generation and applications to channel and flat-plate flows. École Centrale de Lyon; 2016.

[21] Cottet GH, Koumoutsakos P, Salihi MLO. Vortex methods with spatially varying cores. J Comput Phys 2000;162(1):164-85. doi:10.1006/jcph.2000.6531.

[22] Schlichting H, Gersten K, Krause E, Oertel H. Boundary-layer theory, vol. 7. Springer; 1955.

[23] Cousteix J. Turbulence et couche limite. Cepadues editions; 1989

[24] Batchelor GK. The theory of homogeneous turbulence. Cambridge university press; 1953.

[25] Tavoularis S, Bennett JC, Corrsin S. Velocity-derivative skewness in small Reynolds number, nearly isotropic turbulence. J Fluid Mech 1978;88(01):63-9.

[26] Yaglom A, Monin A. Statistical fluid mechanics: mechanics of turbulence, vol. 1. Courier Corporation; 2007. 
[27] Bos WJT, Chevillard L, Scott JF, Rubinstein R. Reynolds number effect on the velocity increment skewness in isotropic turbulence. Phys Fluids (1994-present) 2012;24(1):015108.

[28] Comte-Bellot G, Craya A. Écoulement turbulent entre deux parois parallèles. Service de documentation scientifique et technique de l'armement; 1965.

[29] Burattini P, Lavoie P, Antonia RA. Velocity derivative skewness in isotropic turbulence and its measurement with hot wires. Exp fluids 2008;45(3):523-35.

[30] Hill RJ. Exact second-order structure-function relationships. J Fluid Mechanics 2002;468:317-26.

[31] Antonia RA, Chambers AJ, Satyaprakash BR. Reynolds number dependence of high-order moments of the streamwise turbulent velocity derivative. Boundary-Layer Meteorol 1981;21(2):159-71.

[32] Gylfason A, Ayyalasomayajula S, Warhaft Z. Intermittency, pressure and acceleration statistics from hot-wire measurements in wind-tunnel turbulence. J Fluid Mech 2004;501:213-29.

[33] Ishihara T, Kaneda Y, Yokokawa M, Itakura K, Uno A. Small-scale statistics in high-resolution direct numerical simulation of turbulence: Reynolds number dependence of one-point velocity gradient statistics. J Fluid Mech 2007;592:335-66.
[34] Nicoud F, Ducros F. Subgrid-scale stress modelling based on the square of the velocity gradient tensor. Flow Turbul Combust 1999;62(3):183-200.

[35] Jameson A. Transonic airfoil calculations using the Euler equations. Numerical Methods in Aeronautical Fluid Dynamics. Citeseer; 1982.

[36] Kim J, Moin P, Moser R. Turbulence statistics in fully developed channel flow at low reynolds number. J Fluid Mech 1987:177:133-66.

[37] Wilcox DC. Reassessment of the scale-determining equation for advanced turbulence models. AIAA J 1988;26(11):1299-310.

[38] Lesieur M. Turbulence in fluids, vol. 40. Springer Science \& Business Media; 2012.

[39] Boudet J, Monier JF, Gao F. Implementation of a roughness element to trip transition in large-eddy simulation. J Therm Sci 2015;24(1):30-6.

[40] Jiménez J, Hoyas S, Simens MP, Mizuno Y. Turbulent boundary layers and channels at moderate Reynolds numbers. J Fluid Mech 2010;657:335-60. doi:10. $1017 /$ S0022112010001370. 